\title{
Generalized threshold resummation in inclusive DIS and semi-inclusive electron-positron annihilation
}

\author{
A.A. Almasy, ${ }^{a, 1}$ N.A. Lo Presti ${ }^{b}$ and A. Vogt ${ }^{c}$ \\ ${ }^{a}$ Deutsches Elektronensynchrotron DESY, \\ Platanenallee 6, D-15738 Zeuthen, Germany \\ ${ }^{b}$ Department of Physics, University of Zürich, \\ Winterthurerstrasse 190, CH-8057 Zürich, Switzerland \\ ${ }^{c}$ Department of Mathematical Sciences, University of Liverpool, \\ Liverpool L69 3BX, U.K. \\ E-mail: a.a.almasy@gmail.com, lopresti@physik.uzh.ch, \\ Andreas.Vogt@liverpool.ac.uk
}

ABSTRACT: We present analytic all-order results for the highest three threshold logarithms of the space-like and time-like off-diagonal splitting functions and the corresponding coefficient functions for inclusive deep-inelastic scattering (DIS) and semi-inclusive $e^{+} e^{-}$annihilation. All these results, obtained through an order-by-order analysis of the structure of the corresponding unfactorized quantities in dimensional regularization, can be expressed in terms of the Bernoulli functions introduced by one of us and leading-logarithmic soft-gluon exponentials. The resulting numerical corrections are small for the splitting functions but large for the coefficient functions. In both cases more terms in the threshold expansion need to be determined in order to arrive at quantitatively reliable results.

KeYwords: Resummation, Perturbative QCD

ARXIV EPRINT: 1511.08612

\footnotetext{
${ }^{1}$ Address until 31 August 2013.
} 


\section{Contents}

1 Introduction 1

2 Threshold limits, mass factorization and resummation 2

$\begin{array}{lll}3 & \text { The NNLL corrections in DIS in closed form } & 7\end{array}$

4 NNLL resummation in semi-inclusive $e^{+} e^{-}$annihilation 11

$\begin{array}{llr}5 & \text { Summary and outlook } & 16\end{array}$

\section{Introduction}

Inclusive deep-inelastic lepton-hadron scattering (DIS) and semi-inclusive electron-positron annihilation (SIA) are phenomenologically and theoretically important benchmark processes in perturbative quantum chromodynamics (QCD). Data on their cross sections, respectively expressed in terms of structure functions and fragmentation functions, form a primary source of information on the parton distributions and fragmentation distributions of initial- and final-state hadrons [1]. The calculation of these functions in dimensional regularization is a standard way to determine the splitting functions governing the scale dependence (evolution) of the perturbatively incalculable but process-independent parton and fragmentation distributions. The coefficient functions (partonic cross sections) for DIS and SIA, together with those for inclusive lepton-pair and Higgs-boson production in hadron collisions, facilitate studies of the analytic structure of QCD corrections at and beyond the next-to-next-to-leading order (NNLO) of the renormalization-group improved perturbative expansion [2-16], an accuracy that is harder to achieve for less inclusive quantities.

With the notable exception of the $\overline{\mathrm{MS}}$-scheme quark-quark and gluon-gluon splitting functions in the threshold limit [17-23], all those perturbative quantities receive logarithmically enhanced higher-order corrections near kinematic limits. Depending on the observable and the kinematic region, these logarithms can require an all-order resummation in order to achieve phenomenologically reliable predictions. Knowledge of the endpoint behaviour of splitting or coefficient functions is also useful beyond such situations, e.g., if it can be combined with other partial information such as a finite number of Mellin moments of the splitting function or coefficient function under consideration, for a recent example see ref. [24].

In this article we address the all-order resummation of threshold $(x \rightarrow 1)$ logarithms of the form

$$
(1-x)^{\xi} \ln ^{2 n-n_{0}-\ell}(1-x) \quad \text { with } \quad \xi=0,1 \quad \text { and } \quad \ell=0,1,2
$$


for the $n^{\text {th }}$-order splitting functions and coefficient functions occurring in DIS and SIA. For some coefficient functions the $\xi=0$ terms are subleading to the $\xi=-1$ logarithms which are the subject of the soft-gluon exponentiation (SGE) [25-29] — for the present status in DIS and SIA see refs. [30,31]; for other quantities the $\xi=0$ or even $\xi=1$ terms form the leading contributions. Specifically, we complete the study of DIS in ref. [32] by deriving analytic formulae for the next-to-next-to-leading logarithmic (NNLL) $\ell=2$ corrections and present the corresponding results for SIA. A brief previous account of the latter can be found in ref. [33], see also ref. [34].

The present resummations of DIS and SIA to NNLL accuracy are based on the NNLO fixed-order results, the structure of the unfactorized structure functions and fragmentation functions in $D$ dimensions and the constraints imposed by the all-order mass-factorization formula. It is worthwhile to note that the different phase-space structure of the Drell-Yan process and Higgs production prevents a direct generalization of this approach to these cases beyond the leading-logarithmic $\ell=0$ accuracy of ref. [35]. The reader is referred to refs. [36-40] for an alternative approach (with identical results where both methods are applicable) using physical evolution kernels, which is particularly suited for deriving all- $\xi$ results at a fixed order $n$. For other, partly more formal research on subleading threshold logarithms see refs. [41-50].

\section{Threshold limits, mass factorization and resummation}

The calculations in massless perturbative QCD often take a more compact and transparent form in Mellin- $N$ space, defined through the integral transforms

$$
f(N)=\int_{0}^{1} d x x^{N-1} f(x) \text { and } \quad f(N)=\int_{0}^{1} d x\left(x^{N-1}-1\right) f(x)_{+},
$$

respectively, of integrable functions and of plus-distributions. A main advantage of working in $N$-space is that the ubiquitous (multiple) Mellin convolutions are reduced to simple product, e.g.,

$$
[f \otimes g](x)=\int_{x}^{1} \frac{d y}{y} f(y) g\left(\frac{x}{y}\right) \stackrel{\mathrm{M}}{=} f(N) g(N)
$$

where $\stackrel{\mathrm{M}}{=}$ indicates that the r.h.s. is the Mellin transform of the previous expression. The threshold limit $x \rightarrow 1$ for the scaling variable, e.g., Bjorken- $x$ in DIS, corresponds to the limit $N \rightarrow \infty$. To NNLL accuracy the dictionary between the $x$-space and $N$-space threshold logarithms reads

$$
\begin{aligned}
& \left(\frac{\ln ^{k-1}(1-x)}{1-x}\right)_{+} \stackrel{\mathrm{M}}{=} \frac{(-1)^{k}}{k}\left(\left[S_{1-}(N)\right]^{k}+\frac{1}{2} k(k-1) \zeta_{2}\left[S_{1-}(N)\right]^{k-2}+O\left(\left[S_{1-}(N)\right]^{k-3}\right)\right), \\
& \ln ^{k}(1-x) \stackrel{\mathrm{M}}{=} \frac{(-1)^{k}}{N}\left(\ln ^{k} \tilde{N}+\frac{1}{2} k(k-1) \zeta_{2} \ln ^{k-2} \widetilde{N}+O\left(\ln ^{k-3} \widetilde{N}\right)\right), \\
& (1-x) \ln ^{k}(1-x) \stackrel{\mathrm{M}}{=} \frac{(-1)^{k}}{N^{2}}\left(\ln ^{k} \tilde{N}-k \ln ^{k-1} \widetilde{N}+\frac{1}{2} k(k-1) \zeta_{2} \ln ^{k-2} \widetilde{N}+O\left(\ln ^{k-3} \widetilde{N}\right)\right),
\end{aligned}
$$


where $S_{1-}(N)=\ln \widetilde{N}-1 /(2 N)+O\left(1 / N^{2}\right)$ and $\widetilde{N}=N e^{\gamma_{\mathrm{e}}}$ with Euler's constant $\gamma_{\mathrm{e}} \simeq$ 0.577216 . Terms suppressed by an extra power of $1 / N$ have been included only in the first line of eq. (2.3).

In order to obtain resummed expressions for the splitting and coefficient functions, we address the unfactorized partonic structure functions and fragmentation functions $T_{a, k}$ for $a=2(T), L, \phi$ and $k=\mathrm{q}$, g, where $\phi$ denotes a scalar directly coupling only to gluons, such as the Higgs boson in the limit of a heavy top-quark and negligible other quark masses. These functions depend on $N$, the strong coupling $\alpha_{\mathrm{s}}$ and, in dimensional regularization, $D=4-2 \epsilon$. In contrast to the physical structure functions $F_{2, L, \phi}$ and the transverse, longitudinal and $\phi$-exchange fragmentation functions $F_{T}$ and $F_{L, \phi}^{T}$, the $T_{a, k}$ are parton-level quantities that contain poles in the dimensional regulator $\epsilon$ and that have not been convoluted with the parton or fragmentation distributions.

Dropping all the functional dependences for brevity, these quantities can be factorized as

$$
T_{a, k}=\widetilde{C}_{a, i} Z_{i k}^{(T)}
$$

where the universal transition functions $Z_{i k}^{(T)}$ collect all negative powers of $\epsilon$ and, in the flavour-singlet DIS ('space-like') case, satisfy the equation

$$
-\gamma \equiv P=\frac{d Z}{d \ln Q^{2}} Z^{-1} \quad \text { with } \quad P=\left(\begin{array}{cc}
P_{q q} & P_{q g} \\
P_{g q} & P_{g g}
\end{array}\right),
$$

where $P_{i k}$ are the initial state (space-like) splitting functions; the $D$-dimensional coefficient functions $\widetilde{C}_{a, i}$ are addressed below in eq. (2.12). The final-state fragmentation ('timelike') transition functions $Z_{i k}^{T}$ satisfy an analogous equation with $P_{i k}$ replaced by $P_{k i}^{T}$. In eq. (2.5) we have identified, without loss of information, all scales with the physical scale $Q^{2}$ provided by the (space-like or time-like) momentum $q$ of the exchanged gauge boson or scalar, $Q^{2}=-q^{2}$ in DIS and $Q^{2}=q^{2}$ in SIA. This identification will be used throughout this article for both the renormalization and the mass-factorization scale in the $\overline{\mathrm{MS}}$ scheme.

The (space-like and time-like) splitting functions $P_{i k}$ - for the rest of this section we suppress the superscripts $(T)$ and $T$ - can be expanded in powers of the strong coupling constant $\alpha_{\mathrm{s}}$ as

$$
P_{i k}\left(N, \alpha_{\mathrm{s}}\right)=\sum_{n=0}^{\infty} a_{\mathrm{s}}^{n+1} P_{i k}^{(n)}(N) \quad \text { with } \quad a_{\mathrm{s}} \equiv \frac{\alpha_{\mathrm{s}}}{4 \pi} .
$$

These functions are (in the time-like case: almost, with an uncertainty irrelevant to the present considerations) completely known to NNLO, here the third order in $\alpha_{\mathrm{s}}$ [19-23]. The diagonal (quark-quark and gluon-gluon) splitting functions have a stable form in the large- $N$ limit $[17,18]$,

$$
P_{k k}^{(n-1)}(N)=-A_{k}^{(n)} \ln \tilde{N}+B_{k}^{(n)} \pm C_{k}^{(n)} N^{-1} \ln \tilde{N}+\mathcal{O}\left(N^{-1}\right)
$$

with the $n$-loop quark and gluon cusp anomalous dimensions related by $A_{\mathrm{g}}^{(n)} / A_{\mathrm{q}}^{(n)}=$ $C_{A} / C_{F}$ at $n \leq 3$, a fact often referred to as Casimir scaling. The coefficient of $N^{-1} \ln \tilde{N}$ can be expressed in terms of lower-order cusp anomalous dimensions [18], and differs in sign 
between the DIS and SIA cases. The large- $N$ behaviour of the off-diagonal (quark-gluon and gluon-quark) splitting functions, on the other hand, is characterized by a doublelogarithmic higher-order enhancement,

$$
P_{i \neq k}^{(n)}(x)=\frac{1}{N} \sum_{\ell=0}^{2 n} D_{i k}^{(n, \ell)} \ln ^{2 n-\ell} \tilde{N}+\mathcal{O}\left(\frac{1}{N^{2}} \ln ^{m} \tilde{N}\right),
$$

where the terms with $\ell=0$ form the leading-logarithmic (LL) approximation [51], those with $\ell=1$ the next-to-leading-logarithmic (NLL) contributions etc. Analytic results for the $\ell \leq 2$ coefficients in eq. (2.8) will be presented in sections 4 and 5 below.

After expanding the transition functions in powers of the strong coupling as

$$
Z_{i k}=\sum_{n=1}^{\infty} a_{\mathrm{s}}^{n} Z_{i k}^{(n)}
$$

Eq. (2.5) can be solved order by order in $a_{\mathrm{s}}$. With $\gamma_{n}=-P^{(n)}$ this results in

$$
\begin{aligned}
Z= & +a_{\mathrm{s}} \frac{1}{\epsilon} \gamma_{0}+a_{\mathrm{s}}^{2}\left\{\frac{1}{2 \epsilon^{2}}\left(\gamma_{0}-\beta_{0}\right) \gamma_{0}+\frac{1}{2 \epsilon} \gamma_{1}\right\} \\
& +a_{\mathrm{s}}^{3}\left\{\frac{1}{6 \epsilon^{3}}\left(\gamma_{0}-\beta_{0}\right)\left(\gamma_{0}-2 \beta_{0}\right) \gamma_{0}+\frac{1}{6 \epsilon^{2}}\left[\left(\gamma_{0}-2 \beta_{0}\right) \gamma_{1}+\left(\gamma_{1}-\beta_{1}\right) 2 \gamma_{0}\right]+\frac{1}{3 \epsilon} \gamma_{2}\right\} \\
& +a_{\mathrm{s}}^{4}\left\{\frac{1}{24 \epsilon^{4}}\left(\gamma_{0}-\beta_{0}\right)\left(\gamma_{0}-2 \beta_{0}\right)\left(\gamma_{0}-3 \beta_{0}\right) \gamma_{0}\right. \\
& +\frac{1}{24 \epsilon^{3}}\left[\left(\gamma_{0}-2 \beta_{0}\right)\left(\gamma_{0}-3 \beta_{0}\right) \gamma_{1}+\left(\gamma_{0}-3 \beta_{0}\right)\left(\gamma_{1}-\beta_{1}\right) 2 \gamma_{0}+\left(\gamma_{1}-2 \beta_{1}\right)\left(\gamma_{0}-\beta_{0}\right) 3 \gamma_{0}\right] \\
& \left.+\frac{1}{24 \epsilon^{2}}\left[\left(\gamma_{0}-3 \beta_{0}\right) 2 \gamma_{2}+\left(\gamma_{1}-2 \beta_{1}\right) 3 \gamma_{1}+\left(\gamma_{2}-\beta_{2}\right) 6 \gamma_{0}\right]+\frac{1}{4 \epsilon} \gamma_{3}\right\}+\ldots .
\end{aligned}
$$

Here $\beta_{n}$ are the usual $\mathrm{N}^{n} \mathrm{LO}$ coefficients of the beta function of QCD, with $\beta_{0}=11 / 3 C_{A}-$ $2 / 3 n_{f}$, where $n_{f}$ is the number of effectively massless quark flavours and $C_{A}=n_{\text {colours }}=$ 3 . There is no general closed all-order form of this result; however for the present large$N$ limit an explicit, if still rather lengthy form has been given in eq. (2.7)-(2.13) of ref. [32]. Schematically, the leading-logarithmic behaviour of $Z$ in $N$-space corresponding to eqs. (2.7) and (2.8) is given by

$$
\begin{aligned}
& Z_{k k}^{(n)} \sim\left(1+\epsilon \ln \widetilde{N}+\ldots+\epsilon^{n-1} \ln ^{n-1} \widetilde{N}\right) \epsilon^{-n} \ln ^{n} \widetilde{N} \\
& Z_{i \neq k}^{(n)} \sim\left(1+\epsilon \ln \widetilde{N}+\ldots+\epsilon^{n-1} \ln ^{n-1} \widetilde{N}\right) \epsilon^{-n} N^{-1} \ln ^{n-1} \widetilde{N}
\end{aligned}
$$

The process-dependent $D$-dimensional coefficient functions $\widetilde{C}_{a, i}$ in eq. (2.4) include contributions with all non-negative powers of $\epsilon$. Their expansion in powers of $\alpha_{\mathrm{s}}$ and $\epsilon$ can be written as

$$
\widetilde{C}_{a, i}=\delta_{a \gamma} \delta_{i \mathrm{q}}+\delta_{a \phi} \delta_{i \mathrm{~g}}+\sum_{n=1}^{\infty} a_{\mathrm{s}}^{n} \sum_{k=0}^{\infty} \epsilon^{k} c_{a, i}^{(n, k)} .
$$

Here the index $\gamma$ of the Kronecker-delta indicates that $\delta_{a \gamma}$ is equal to one if $a=2$ in DIS and $a=T$ in SIA, and zero for $a=\phi$ or $L$. The $\epsilon$-independent contributions, $c_{a, i}^{(n, 0)} \equiv$ 
$c_{a, i}^{(n)}$, are the $n^{\text {th }}$-order coefficient functions entering the physical structure functions and fragmentation functions.

The quark coefficient functions for the gauge-boson-exchange structure and fragmentation functions $F_{2, T}$ and the gluon coefficient function for the scalar-exchange structure and fragmentation function $F_{\phi}$, also referred to as 'diagonal' coefficient functions, are dominated in the large- $N$ limit by Mellin-transformed plus-distributions with a the double-logarithmic enhancement,

$$
c_{a, k}^{(n)}(x)=\sum_{\ell=0}^{2 n} D_{a, k}^{(n, \ell)} \ln ^{2 n-\ell} \widetilde{N}+\frac{1}{N} \sum_{\ell=0}^{2 n-1} E_{a, k}^{(n, \ell)} \ln ^{2 n-1-\ell} \tilde{N}+\mathcal{O}\left(\frac{1}{N^{2}} \ln ^{m} \tilde{N}\right)
$$

for $\{a, k\}=\{2, q\},\{T, q\}$ and $\{\phi, g\}$. The first sum in eq. (2.13) includes the contributions that are resummed by the soft-gluon exponentiation [25-29], with the coefficients $D_{a, k}^{(n, 0)}$ $\ldots D_{a, k}^{(n, 2 n-2)}$ at order $n$ fixed by lower-order information. At present the coefficients of the six highest logarithms are known analytically, and for the seventh only the (numerically small) contribution from the four-loop cusp anomalous dimension is missing; see ref. [30] and ref. [31] for the respective gauge-boson exchange DIS and SIA results.

Complete all-order results for the LL, NLL and NNLL $(\ell \leq 2)$ coefficients $E_{2, q}^{(n, \ell)}$ and $E_{T, q}^{(n, \ell)}$ in eq. (2.13) were derived in ref. [37] from a conjecture on the respective physical evolution kernels. In the present approach, we were able to verify those results (and hence the underlying conjecture) and to extend them to the $\ell=3$ coefficients, thus fixing the corresponding unknown coefficients in ref. [37] as $\xi_{\mathrm{DIS}_{4}}=\xi_{\mathrm{SIA}_{4}}$ [34]. This agrees with the result obtained in ref. [49].

The remaining coefficient functions for the observables in eq. (2.13) start only at order $\alpha_{\mathrm{s}}$ and can be considered as 'off-diagonal' quantities (the off-diagonal splitting functions arise from their unfactorized counterparts). Their leading large- $N$ behaviour is completely analogous to the subleading $1 / N$ contributions in eq. (2.13), viz

$$
c_{a, k}^{(n)}(x)=\frac{1}{N} \sum_{\ell=0}^{2 n-1} D_{a, k}^{(n, \ell)} \ln ^{2 n-1-\ell} \tilde{N}+\mathcal{O}\left(\frac{1}{N^{2}} \ln ^{m} \tilde{N}\right)
$$

for $\{a, k\}=\{2, g\},\{T, g\}$ and $\{\phi, q\}$. Besides their splitting-function counterparts in eq. (2.8) above, the all-order determination of the $l \leq 2$ coefficients constitutes in eq. (2.14) a main objective of this article; the corresponding DIS and SIA results can be found in sections 4 and 5 .

Finally the large- $N$ expansion of the $n^{\text {th }}$-order coefficient functions for the structure function $F_{L}$ and the fragmentation function $F_{L}^{T}$ have the form, for $k=q, g$,

$$
c_{L, k}^{(n)}(x)=\frac{1}{N^{1+\delta_{k g}}} \sum_{\ell=0}^{2 n-2} D_{L, k}^{(n, \ell)} \ln ^{2 n-2-\ell} \tilde{N}+\mathcal{O}\left(\frac{1}{N^{2+\delta_{k g}}} \ln ^{m} \tilde{N}\right) .
$$

In the quark cases our present calculations verify the physical-kernel based results of refs. $[36,37]$ for the coefficients up to NNLL accuracy $(l \leq 2)$, but cannot add to those 
results. The closed form of the NNLL resummation for the case of DIS has been given already in eq. (6.3) of ref. [32]; the corresponding SIA result will be presented in section 5.

The $k>0$ coefficients $c_{a, i}^{(n, k)}$ in the $\epsilon$-expansion of the $D$-dimensional coefficient function in eq. (2.12) are enhanced by factors $\ln ^{k} \widetilde{N}$ with respect to the four-dimensional coefficient functions discussed in eqs. (2.13)-(2.15), i.e., the pattern for the non-negative powers of $\epsilon$ is the same as in eq. (2.11) for the $1 / \epsilon$ poles. Consequently the unfactorized structure functions and fragmentation functions $T_{a, k}$ in eq. (2.4) exhibit the same structure over all powers of $\epsilon$.

Disregarding the logarithms and (except for $T_{L, g}$ ) terms $\widetilde{C}_{a, i} Z_{i k}$ that contribute only at order $1 / N^{2}$, the large- $N$ behaviour of the unfactorized structure functions and fragmentation functions can be summarized as follows, with $\gamma=2$ in DIS and $\gamma=T$ in SIA,

$$
\begin{aligned}
T_{\gamma, q} & \simeq \widetilde{C}_{\gamma, q} Z_{q q} \sim \mathcal{O}(1) \quad \rightarrow T_{\gamma, g}=\widetilde{C}_{\gamma, q} Z_{q g}+\widetilde{C}_{\gamma, g} Z_{g g} \sim \mathcal{O}(1 / N), \\
T_{\phi, g} & \simeq \widetilde{C}_{\phi, g} Z_{g g} \sim \mathcal{O}(1) \quad \rightarrow T_{\phi, q}=\widetilde{C}_{\phi, g} Z_{g q}+\widetilde{C}_{\phi, q} Z_{q q} \sim \mathcal{O}(1 / N), \\
T_{L, q} \simeq \widetilde{C}_{L, q} Z_{q q} \sim \mathcal{O}(1 / N) \rightarrow T_{L, g} & =\widetilde{C}_{L, q} Z_{q g}+\widetilde{C}_{L, g} Z_{g g} \sim \mathcal{O}\left(1 / N^{2}\right) .
\end{aligned}
$$

Here the arrows indicate that the resummation of the quantities on the left-hand-side, including positive powers of $\epsilon$, is needed in order to extract the all-order 'off-diagonal' coefficient functions.

Once the unfactorized structure function is known at order $a_{\mathrm{s}}^{n}$, it is possible to extract the coefficient function $c_{a, i}^{(n, 0)}$, provided that the lower-order contributions $c_{a, i}^{(m, k)}$ are known to a sufficiently high power of $\epsilon$. In particular, the calculation of $T_{a}$ to order $a_{\mathrm{s}}^{\ell \leq n}$ and $\epsilon^{n-\ell}$ is required for the extraction of the coefficient function at order $a_{\mathrm{s}}^{n}$. On the other hand, one can see from eq. (2.10) that a full $\mathrm{N}^{n-1} \mathrm{LO}$ result completely fixes the highest $n$ powers of $1 / \epsilon$ to all orders in $a_{\mathrm{s}}$. In order to be able to extract the splitting and coefficient functions from the unfactorized structure function and fragmentation functions at all orders (at the logarithmic accuracy under consideration), it is thus necessary to consider the $D$ dimensional coefficient functions at all powers of $\epsilon$.

It was noted in ref. [32] that the $a_{\mathrm{s}}^{n}$ contributions to the unfactorized structure functions $T_{2, g}, T_{\phi, q}$ and $T_{L, k}$ in Mellin space can be written as

$T_{a, k}^{(n)}(N)=\frac{1}{N^{1+\delta_{a L} \delta_{k g}} \epsilon^{2 n-1-\delta_{a L}}} \sum_{i=0}^{n-1}\left(A_{a, k}^{(n, i)}+\epsilon B_{a, k}^{(n, i)}+\epsilon^{2} C_{a, k}^{(n, i)}+\ldots\right) \exp (\epsilon(n-i) \ln N)$.

The fact that the right-hand-side features double poles in $\epsilon$, whereas the mass-factorization formula ensures that only single poles appear in the unfactorized structure function, imposes constraints on the coefficients $A_{a, k}^{(n, i)}, B_{a, k}^{(n, i)}$ and $C_{a, k}^{(n, i)}$. In the large- $N$ limit, once the exponential on the r.h.s. is expanded, only the leading logarithmic (LL) coefficients $A_{a, k}^{(n, i)}$ appear in all the vanishing $\epsilon^{-2 n+1}, \ldots, \epsilon^{-n-1}$ terms, so we immediately have $n-1$ relations for these coefficients. The next-to-leading logarithmic (NLL) coefficients $B_{a, k}^{(n, i)}$ will appear in all double-pole terms except the one proportional to $\epsilon^{-2 n+1}$, resulting in $n-2$ equations; at next-to-next-to-leading logarithmic (NNLL) level there are $n-3$ relations for the coefficients $C_{a, k}^{(n, i)}$ and so on. In general, the cancellation of the double poles 
in $\epsilon$ provides $n-1-m$ relations between the $n \mathrm{~N}^{m} \mathrm{LL}$ coefficients. The first $k+1$ powers of $\epsilon^{-1}$ with non-vanishing coefficients are fixed by a full $\mathrm{N}^{k} \mathrm{LO}$ calculation, as discussed at the end of the previous section, leading to a total of $n-m+k$ relations. Since at order $a_{\mathrm{s}}^{n}$ each linear system has $n$ unknowns (notice the sum over the index $i$ in eq. (2.17)), the coefficients up to the $\mathrm{N}^{k} \mathrm{LL}$ terms are fixed in terms of the $\mathrm{N}^{k} \mathrm{LO}$ results. The $\mathrm{N}^{m} \mathrm{LL}$ coefficients with $m<k$ are over-constrained to all orders, providing a check on the correctness of eq. (2.17). The same holds for $m=k$ beyond order $k$.

Having determined the coefficients of eq. (2.17), the mass-factorization formula (2.4), together with the all-order solution of eq. (2.5) in the large- $N$ limit, see eqs. (2.7)-(2.13) of ref. [32], allows the iterative determination of the coefficients $D_{i k}^{(n, \ell \leq 2)}$ in eq. (2.8) and $D_{a, k}^{(n, \ell \leq 2)}$ in (2.14) and (2.15) to, in principle, any order in $\alpha_{\mathrm{s}}$. It may be worthwhile to note that the all-order expressions for $Z_{i k}^{(n)}$ are not a superfluous luxury: the results of the mass factorization are required to a very high order, beyond what can be easily achieved by an order-by-order brute-force solution of eq. (2.5), for the reconstruction of the NNLL analytic forms presented in the next two sections.

\section{The NNLL corrections in DIS in closed form}

In ref. [51] it was found that the LL contributions to the resummed off-diagonal splitting and coefficient functions can be expressed in a closed form in terms of an apparently new function $\mathcal{B}_{0}$,

$$
\begin{aligned}
\mathcal{B}_{0}(x)=\sum_{n=0}^{\infty} \frac{B_{n}}{(n !)^{2}} x^{n} & =1-\frac{x}{2}-\sum_{n=1}^{\infty} \frac{(-1)^{n}}{[(2 n) !]^{2}}\left|B_{2 n}\right| x^{2 n} \\
& =1-\frac{x}{2}-2 \sum_{n=1}^{\infty} \frac{(-1)^{n}}{(2 n) !} \zeta_{2 n}\left(\frac{x}{2 \pi}\right)^{2 n}
\end{aligned}
$$

Here $B_{n}$ are the Bernoulli numbers as normalized in ref. [52]; $\zeta_{n}$ denotes Riemann's $\zeta$-function. Due to $\zeta_{n} \rightarrow 1$ for $n \rightarrow \infty$, the Taylor series (3.1) absolutely converges for all values of $x$.

This resummation was extended to the NLL and NNLL contributions to the splitting functions for the evolution of parton distributions and the coefficients functions for inclusive DIS in ref. [32]. However, with the exception of the longitudinal structure function, closed forms were found only for the NLL corrections. Besides eq. (3.1), these expressions involve the generalizations

$$
\mathcal{B}_{k}(x)=\sum_{n=0}^{\infty} \frac{B_{n}}{n !(n+k) !} x^{n}, \quad \mathcal{B}_{-k}(x)=\sum_{n=k}^{\infty} \frac{B_{n}}{n !(n-k) !} x^{n}
$$

which are related to $\mathcal{B}_{0}$ by

$$
\frac{d^{k}}{d x^{k}}\left(x^{k} \mathcal{B}_{k}\right)=\mathcal{B}_{0}, \quad \frac{d^{k}}{d x^{k}} \mathcal{B}_{0}=\frac{1}{x^{k}} \mathcal{B}_{-k} .
$$

Specifically, the NLL terms can be expressed by $\mathcal{B}_{k}$ with $-2 \leq k \leq 1$; plots of these functions can be found in figure 1 of ref. [32]. On the other hand, the NNLL corrections for 
$P_{q g}, P_{q g}, C_{2, g}$ and $C_{\phi, q}$ were only given via tables to order $\alpha_{\mathrm{s}}^{18}$ for the off-diagonal splitting functions and to order $\alpha_{\mathrm{s}}^{12}$ for the corresponding coefficient functions.

By extending the calculations to a considerably higher order than before, and thus generating an over-constrained system of linear equations for a suitably general ansatz, we have been able to derive the hitherto missing closed forms. They are much more complicated than their LL and NLL counterparts, but involve the same ingredients: the functions $\mathcal{B}_{k}$, for the coefficient functions in combination with the LL exponentials for the soft-gluon resummation of $C_{2, q}$ and $C_{\phi, g}$.

The large- $N$ space-like gluon-quark and quark-gluon splitting functions read, at NNLL accuracy,

$$
\begin{aligned}
N P_{\mathrm{qg}}^{\mathrm{S}}\left(N, \alpha_{\mathrm{s}}\right)= & 2 n_{f} a_{\mathrm{s}} \mathcal{B}_{0} \\
& +n_{f} a_{\mathrm{s}}^{2} \ln \tilde{N}\left\{\left(6 C_{F}-\beta_{0}\right)\left[\mathcal{B}_{1}+2 \tilde{a}_{\mathrm{s}}^{-1} \mathcal{B}_{-1}\right]+\beta_{0} \tilde{a}_{\mathrm{s}}^{-1} \mathcal{B}_{-2}\right\} \\
& +\frac{n_{f} a_{\mathrm{s}}^{2}}{48 C_{A F}}\left\{108 C_{F}^{2}\left[2 \tilde{a}_{\mathrm{s}} \mathcal{B}_{2}-4 \mathcal{B}_{1}+5 \mathcal{B}_{0}+2 \tilde{a}_{\mathrm{s}}^{-1} \mathcal{B}_{-1}+4 \tilde{a}_{\mathrm{s}}^{-1} \mathcal{B}_{-2}\right]\right. \\
& -36 \beta_{0} C_{F}\left[\tilde{a}_{\mathrm{s}} \mathcal{B}_{2}-3 \mathcal{B}_{1}+4 \mathcal{B}_{0}-\mathcal{B}_{-1}+2 \tilde{a}_{\mathrm{s}}^{-1} \mathcal{B}_{-1}+\tilde{a}_{\mathrm{s}}^{-1} \mathcal{B}_{-2}-2 \tilde{a}_{\mathrm{s}}^{-1} \mathcal{B}_{-3}\right] \\
& +\beta_{0}^{2}\left[2 \tilde{a}_{\mathrm{s}} \mathcal{B}_{2}-12 \mathcal{B}_{1}+12 \mathcal{B}_{0}-6 \mathcal{B}_{-1}-12 \tilde{a}_{\mathrm{s}}^{-1} \mathcal{B}_{-2}-4 \tilde{a}_{\mathrm{s}}^{-1} \mathcal{B}_{-3}+3 \tilde{a}_{\mathrm{s}}^{-1} \mathcal{B}_{-4}\right] \\
& +80 C_{A F} \beta_{0}\left[\tilde{a}_{\mathrm{s}} \mathcal{B}_{2}-4 \mathcal{B}_{1}+4 \mathcal{B}_{0}+\mathcal{B}_{-1}\right] \\
& -32 C_{A F} C_{F}\left[\left(19-3 \zeta_{2}\right) \tilde{a}_{\mathrm{s}} \mathcal{B}_{2}-34 \mathcal{B}_{1}+\left(13+6 \zeta_{2}\right) \mathcal{B}_{0}-\left(2-3 \zeta_{2}\right) \mathcal{B}_{-1}\right] \\
& \left.+32 C_{A F}^{2}\left[\left(2+3 \zeta_{2}\right) \tilde{a}_{\mathrm{s}} \mathcal{B}_{2}+4\left(1+3 \zeta_{2}\right) \mathcal{B}_{1}+2\left(1-6 \zeta_{2}\right) \mathcal{B}_{0}+\left(2-3 \zeta_{2}\right) \mathcal{B}_{-1}\right]\right\}
\end{aligned}
$$

(this expression was already presented in ref. [34]) and

$$
\begin{aligned}
N P_{\mathrm{gq}}^{\mathrm{S}} & \left(N, \alpha_{\mathrm{s}}\right)=2 C_{F} a_{\mathrm{s}} \overline{\mathcal{B}}_{0} \\
& +C_{F} a_{\mathrm{s}}^{2} \ln \tilde{N}\left\{\left(14 C_{F}-8 C_{A}-\beta_{0}\right) \overline{\mathcal{B}}_{1}+6\left(2 C_{F}-\beta_{0}\right) \tilde{a}_{\mathrm{s}}^{-1} \overline{\mathcal{B}}_{-1}-\beta_{0} \tilde{a}_{\mathrm{s}}^{-1} \overline{\mathcal{B}}_{-2}\right\} \\
& +\frac{C_{F} a_{\mathrm{s}}^{2}}{48 C_{A F}}\left\{108 C_{F}^{2}\left[2 \tilde{a}_{\mathrm{s}} \overline{\mathcal{B}}_{2}-8 \overline{\mathcal{B}}_{1}+7 \overline{\mathcal{B}}_{0}+2 \tilde{a}_{\mathrm{s}}^{-1} \overline{\mathcal{B}}_{-1}+4 \tilde{a}_{\mathrm{s}}^{-1} \overline{\mathcal{B}}_{-2}\right]\right. \\
& -36 \beta_{0} C_{F}\left[\tilde{a}_{\mathrm{s}} \overline{\mathcal{B}}_{2}-19 \overline{\mathcal{B}}_{1}+16 \overline{\mathcal{B}}_{0}+\overline{\mathcal{B}}_{-1}+6 \tilde{a}_{\mathrm{s}}^{-1} \overline{\mathcal{B}}_{-1}+15 \tilde{a}_{\mathrm{s}}^{-1} \overline{\mathcal{B}}_{-2}+2 \tilde{a}_{\mathrm{s}}^{-1} \overline{\mathcal{B}}_{-3}\right] \\
& +\beta_{0}^{2}\left[2 \tilde{a}_{\mathrm{s}} \overline{\mathcal{B}}_{2}-108 \overline{\mathcal{B}}_{1}+84 \overline{\mathcal{B}}_{0}+6 \overline{\mathcal{B}}_{-1}+\tilde{a}_{\mathrm{s}}^{-1}\left(48 \overline{\mathcal{B}}_{-1}+156 \overline{\mathcal{B}}_{-2}+44 \overline{\mathcal{B}}_{-3}+3 \overline{\mathcal{B}}_{-4}\right)\right] \\
& -32 C_{A F} \beta_{0}\left[\tilde{a}_{\mathrm{s}} \overline{\mathcal{B}}_{2}-7 \overline{\mathcal{B}}_{1}-9 \overline{\mathcal{B}}_{0}-4 \overline{\mathcal{B}}_{-1}\right] \\
& +32 C_{A F} C_{F}\left[\left(10-3 \zeta_{2}\right) \tilde{a}_{\mathrm{s}} \overline{\mathcal{B}}_{2}-25 \overline{\mathcal{B}}_{1}+3\left(1-2 \zeta_{2}\right) \overline{\mathcal{B}}_{0}+\left(2-3 \zeta_{2}\right) \overline{\mathcal{B}}_{-1}\right] \\
& \left.-32 C_{A F}^{2}\left[\left(2-9 \zeta_{2}\right) \tilde{a}_{\mathrm{s}} \overline{\mathcal{B}}_{2}-4\left(5+3 \zeta_{2}\right) \overline{\mathcal{B}}_{1}+6\left(1+2 \zeta_{2}\right) \overline{\mathcal{B}}_{0}-\left(2-3 \zeta_{2}\right) \overline{\mathcal{B}}_{-1}\right]\right\}
\end{aligned}
$$


with the shorthand notations

$$
\tilde{a}_{\mathrm{s}} \equiv 4 a_{\mathrm{s}} C_{A F} \ln ^{2} \tilde{N}, \quad C_{A F} \equiv C_{A}-C_{F} .
$$

Further, we have suppressed everywhere the argument of the $\mathcal{B}_{k}$ functions and used $\mathcal{B}_{k} \equiv$ $\mathcal{B}_{k}\left(\tilde{a}_{\mathrm{s}}\right)$ and $\overline{\mathcal{B}}_{k} \equiv \mathcal{B}_{k}\left(-\tilde{a}_{\mathrm{s}}\right)$. The respective first lines in eqs. (3.4) and (3.5) represent the LL result [51], the second lines the NLL result [32] and the remaining parts are the new NNLL expressions.

The resummed expressions for the gluon coefficient functions for $F_{2}$ and the quark coefficient function for $F_{\phi}$ at NNLL accuracy read

$$
\begin{aligned}
& N C_{2, \mathrm{~g}}\left(N, \alpha_{\mathrm{s}}\right)=\frac{n_{f}}{2 C_{A F} \ln \widetilde{N}}\left[\mathcal{B}_{0} \mathcal{E}_{F}-\mathcal{E}_{A}\right]+\frac{n_{f}\left(\beta_{0}-3 C_{F}\right)}{8 C_{A F}^{2} \ln ^{2} \widetilde{N}}\left[\mathcal{B}_{0} \mathcal{E}_{F}-\mathcal{E}_{A}\right] \\
& +\frac{n_{f} a_{\mathrm{s}}}{4 C_{A F}}\left\{6 C_{F}\left[\left(\mathcal{B}_{1}+\mathcal{B}_{0}+2 \tilde{a}_{\mathrm{s}}^{-1} \mathcal{B}_{-1}\right) \mathcal{E}_{F}-2 \mathcal{E}_{A}\right]-8 C_{A F} \mathcal{E}_{A}-\beta_{0}\left[\left(\mathcal{B}_{1}\right.\right.\right. \\
& \left.\left.\left.+4 \tilde{a}_{\mathrm{s}}^{-1} \mathcal{B}_{-1}-\tilde{a}_{\mathrm{s}}^{-1} \mathcal{B}_{-2}\right) \mathcal{E}_{F}-\mathcal{E}_{A}\right]\right\}+\frac{n_{f} a_{\mathrm{s}}^{2} \beta_{0} \ln ^{2} \widetilde{N}}{3 C_{A F}}\left[C_{F} \mathcal{B}_{0} \mathcal{E}_{F}-C_{A} \mathcal{E}_{A}\right] \\
& +\frac{n_{f}}{32 C_{A F}^{3} \ln ^{3} \widetilde{N}}\left\{\beta_{0}^{2}\left[\frac{1}{3} \mathcal{B}_{-3}+\frac{1}{8} \mathcal{B}_{-4}\right] \mathcal{E}_{F}-\beta_{0}\left(\beta_{0}-3 C_{F}\right)\left[\mathcal{B}_{-2}+\mathcal{B}_{-3}\right] \mathcal{E}_{F}\right. \\
& \left.+\left(\beta_{0}-3 C_{F}\right)^{2}\left[\left(\mathcal{B}_{0}-\mathcal{B}_{-1}+2 \mathcal{B}_{-2}\right) \mathcal{E}_{F}-\mathcal{E}_{A}\right]\right\} \\
& +\frac{n_{f} a_{\mathrm{s}}}{96 C_{A F}^{2} \ln \widetilde{N}}\left\{-3 \beta_{0}^{2}\left[\left(4 \mathcal{B}_{1}-4 \mathcal{B}_{0}+\mathcal{B}_{-1}\right) \mathcal{E}_{F}-2 \mathcal{E}_{A}\right]\right. \\
& -54 C_{F}^{2}\left[\left(6 \mathcal{B}_{1}-3 \mathcal{B}_{0}-4 \mathcal{B}_{-1}\right) \mathcal{E}_{F}-4 \mathcal{E}_{A}\right]-40 \beta_{0} C_{A F}\left[\left(4 \mathcal{B}_{1}-2 \mathcal{B}_{0}\right.\right. \\
& \left.\left.-\mathcal{B}_{-1}\right) \mathcal{E}_{F}-2 \mathcal{E}_{A}\right]+18 \beta_{0} C_{F}\left[\left(6 \mathcal{B}_{1}-4 \mathcal{B}_{0}-3 \mathcal{B}_{-1}+\mathcal{B}_{-2}\right) \mathcal{E}_{F}-4 \mathcal{E}_{A}\right] \\
& +16 C_{F} C_{A F}\left[\left(34 \mathcal{B}_{1}-2\left(22+3 \zeta_{2}\right) \mathcal{B}_{0}+\left(2-3 \zeta_{2}\right) \mathcal{B}_{-1}\right) \mathcal{E}_{F}+2\left(5+3 \zeta_{2}\right) \mathcal{E}_{A}\right] \\
& \left.+16 C_{A F}^{2}\left[\left(2\left(1+3 \zeta_{2}\right)\left(2 \mathcal{B}_{1}-\mathcal{B}_{0}\right)+\left(2-3 \zeta_{2}\right) \mathcal{B}_{-1}\right) \mathcal{E}_{F}-2\left(1+3 \zeta_{2}\right) \mathcal{E}_{A}\right]\right\} \\
& +\frac{n_{f} a_{\mathrm{s}}^{2} \ln \tilde{N}}{24 C_{A F}^{2}}\left\{\beta_{0}^{2} C_{F}\left[\left(2 \mathcal{B}_{0}-4 \mathcal{B}_{-1}+\mathcal{B}_{-2}\right) \mathcal{E}_{F}-2 \mathcal{E}_{A}\right]\right. \\
& -6 \beta_{0} C_{F}^{2}\left[\left(\mathcal{B}_{0}-2 \mathcal{B}_{-1}\right) \mathcal{E}_{F}-\mathcal{E}_{A}\right]+\beta_{0}^{2} C_{A F}\left[\mathcal{B}_{2} \mathcal{E}_{F}-\mathcal{E}_{A}\right] \\
& -2 \beta_{0} C_{F} C_{A F}\left[\left(9 \mathcal{B}_{2}+9 \mathcal{B}_{1}-29 \mathcal{B}_{0}\right) \mathcal{E}_{F}+17 \mathcal{E}_{A}\right] \\
& +2 C_{F}^{2} C_{A F}\left[\left(54\left(\mathcal{B}_{2}+\mathcal{B}_{1}\right)+\left(43-24 \zeta_{2}\right) \mathcal{B}_{0}\right) \mathcal{E}_{F}-4\left(31-6 \zeta_{2}\right) \mathcal{E}_{A}\right] \\
& +40 \beta_{0} C_{A F}^{2}\left[\mathcal{B}_{2} \mathcal{E}_{F}-\mathcal{E}_{A}\right]+16 C_{A F}^{3}\left[\left(2+3 \zeta_{2}\right) \mathcal{B}_{2} \mathcal{E}_{F}-\left(20-9 \zeta_{2}\right) \mathcal{E}_{A}\right]
\end{aligned}
$$




$$
\begin{aligned}
& \left.-16 C_{F} C_{A F}^{2}\left[\left(\left(19-3 \zeta_{2}\right) \mathcal{B}_{2}-\left(2-3 \zeta_{2}\right) \mathcal{B}_{0}\right) \mathcal{E}_{F}+4\left(7-3 \zeta_{2}\right) \mathcal{E}_{A}\right]\right\} \\
& +\frac{n_{f} a_{\mathrm{s}}^{3} \beta_{0} \ln ^{3} \tilde{N}}{6 C_{A F}}\left\{-\beta_{0} C_{F}\left(\mathcal{B}_{1}-\mathcal{B}_{0}\right) \mathcal{E}_{F}-8 C_{A F}^{2} \mathcal{E}_{A}-20 C_{F} C_{A F} \mathcal{E}_{A}\right. \\
& \left.+6 C_{F}^{2}\left[\left(\mathcal{B}_{1}+\mathcal{B}_{0}\right) \mathcal{E}_{F}-2 \mathcal{E}_{A}\right]\right\}+\frac{n_{f} a_{\mathrm{s}}^{4} \beta_{0}^{2} \ln ^{5} \tilde{N}}{9 C_{A F}}\left[C_{F}^{2} \mathcal{B}_{0} \mathcal{E}_{F}-C_{A}^{2} \mathcal{E}_{A}\right]
\end{aligned}
$$

and

$$
\begin{aligned}
& N C_{\phi, \mathrm{q}}\left(N, \alpha_{\mathrm{s}}\right)=-\frac{C_{F}}{2 C_{A F} \ln \widetilde{N}}\left[\mathcal{E}_{A} \overline{\mathcal{B}}_{0}-\mathcal{E}_{F}\right]-\frac{C_{F}\left(\beta_{0}-3 C_{F}\right)}{8 C_{A F}^{2} \ln ^{2} \widetilde{N}}\left[\mathcal{E}_{A} \overline{\mathcal{B}}_{0}-\mathcal{E}_{F}\right] \\
& -\frac{C_{F} a_{\mathrm{s}}}{4 C_{A F}}\left\{6 C_{F}\left[\mathcal{E}_{A}\left(\overline{\mathcal{B}}_{1}+2 \tilde{a}_{\mathrm{s}}^{-1} \overline{\mathcal{B}}_{-1}\right)-\mathcal{E}_{F}\right]-4 C_{A F}\left[2 \mathcal{E}_{A} \overline{\mathcal{B}}_{1}-3 \mathcal{E}_{F}\right]\right. \\
& \left.-\beta_{0}\left[\mathcal{E}_{A}\left(\overline{\mathcal{B}}_{1}-2 \overline{\mathcal{B}}_{0}+4 \tilde{a}_{\mathrm{s}}^{-1} \overline{\mathcal{B}}_{-1}+\tilde{a}_{\mathrm{s}}^{-1} \overline{\mathcal{B}}_{-2}\right)+\mathcal{E}_{F}\right]\right\} \\
& -\frac{C_{F} a_{\mathrm{s}}^{2} \beta_{0} \ln ^{2} \tilde{N}}{3 C_{A F}}\left[C_{A} \mathcal{E}_{A} \overline{\mathcal{B}}_{0}-C_{F} \mathcal{E}_{F}\right]-\frac{C_{F}}{32 \ln ^{3} \widetilde{N} C_{A F}^{3}}\left\{\beta_{0}^{2}\left(\frac{1}{3} \overline{\mathcal{B}}_{-3}+\frac{1}{8} \overline{\mathcal{B}}_{-4}\right) \mathcal{E}_{A}\right. \\
& \left.+\beta_{0}\left(\beta_{0}-3 C_{F}\right)\left(\overline{\mathcal{B}}_{-2}+\overline{\mathcal{B}}_{-3}\right) \mathcal{E}_{A}+\left(\beta_{0}-3 C_{F}\right)^{2}\left[\left(\overline{\mathcal{B}}_{0}-\overline{\mathcal{B}}_{-1}+2 \overline{\mathcal{B}}_{-2}\right) \mathcal{E}_{A}-\mathcal{E}_{F}\right]\right\} \\
& +\frac{C_{F} a_{\mathrm{s}}}{96 C_{A F}^{2} \ln \widetilde{N}}\left\{3 \beta_{0}^{2}\left[\left(20 \overline{\mathcal{B}}_{1}-16 \overline{\mathcal{B}}_{0}+7 \overline{\mathcal{B}}_{-1}+2 \overline{\mathcal{B}}_{-2}\right) \mathcal{E}_{A}-2 \mathcal{E}_{F}\right]\right. \\
& +54 C_{F}^{2}\left[\left(10 \overline{\mathcal{B}}_{1}-7 \overline{\mathcal{B}}_{0}\right) \mathcal{E}_{A}-2 \mathcal{E}_{F}\right]-18 \beta_{0} C_{F}\left[\left(22 \overline{\mathcal{B}}_{1}-16 \overline{\mathcal{B}}_{0}+3 \overline{\mathcal{B}}_{-1}\right) \mathcal{E}_{A}\right. \\
& \left.-4 \mathcal{E}_{F}\right]-32 \beta_{0} C_{A F}\left[\left(2 \overline{\mathcal{B}}_{1}+3 \overline{\mathcal{B}}_{0}+2 \overline{\mathcal{B}}_{-1}\right) \mathcal{E}_{A}-5 \mathcal{E}_{F}\right] \\
& +16 C_{F} C_{A F}\left[\left(16 \overline{\mathcal{B}}_{1}-3\left(1-2 \zeta_{2}\right) \overline{\mathcal{B}}_{0}-\left(2-3 \zeta_{2}\right) \overline{\mathcal{B}}_{-1}\right) \mathcal{E}_{A}-\left(13+6 \zeta_{2}\right) \mathcal{E}_{F}\right] \\
& \left.-16 C_{A F}^{2}\left[\left(4\left(5+3 \zeta_{2}\right) \overline{\mathcal{B}}_{1}-6\left(1+2 \zeta_{2}\right) \overline{\mathcal{B}}_{0}+\left(2-3 \zeta_{2}\right) \overline{\mathcal{B}}_{-1}\right) \mathcal{E}_{A}-14 \mathcal{E}_{F}\right]\right\} \\
& +\frac{C_{F} a_{\mathrm{s}}^{2} \ln \tilde{N}}{24 C_{A F}^{2}}\left\{-2 \beta_{0} C_{F} C_{A F}\left[\left(-9 \overline{\mathcal{B}}_{2}+18 \overline{\mathcal{B}}_{1}+17 \overline{\mathcal{B}}_{0}+6 \overline{\mathcal{B}}_{-1}\right) \mathcal{E}_{A}-38 \mathcal{E}_{F}\right]\right. \\
& +6 \beta_{0} C_{F}^{2}\left[\left(\overline{\mathcal{B}}_{0}-2 \overline{\mathcal{B}}_{-1}\right) \mathcal{E}_{A}-\mathcal{E}_{F}\right]-\beta_{0}^{2} C_{F}\left[\left(2 \overline{\mathcal{B}}_{0}-4 \overline{\mathcal{B}}_{-1}-\overline{\mathcal{B}}_{-2}\right) \mathcal{E}_{A}-2 \mathcal{E}_{F}\right] \\
& -\beta_{0}^{2} C_{A F}\left[\left(\overline{\mathcal{B}}_{2}-6 \overline{\mathcal{B}}_{1}+14 \overline{\mathcal{B}}_{0}-4 \overline{\mathcal{B}}_{-1}-\overline{\mathcal{B}}_{-2}\right) \mathcal{E}_{A}-5 \mathcal{E}_{F}\right] \\
& -2 C_{F}^{2} C_{A F}\left[\left(54 \overline{\mathcal{B}}_{2}+8\left(2-3 \zeta_{2}\right) \overline{\mathcal{B}}_{0}\right) \mathcal{E}_{A}-\left(43-24 \zeta_{2}\right) \mathcal{E}_{F}\right] \\
& +8 C_{A F}^{3}\left[\left(2\left(2-9 \zeta_{2}\right) \overline{\mathcal{B}}_{2}-2\left(2-3 \zeta_{2}\right) \overline{\mathcal{B}}_{0}\right) \mathcal{E}_{A}+12\left(1+2 \zeta_{2}\right) \mathcal{E}_{F}\right]
\end{aligned}
$$




$$
\begin{aligned}
& -8 C_{F} C_{A F}^{2}\left[\left(\left(20-6 \zeta_{2}\right) \overline{\mathcal{B}}_{2}+4\left(2-3 \zeta_{2}\right) \overline{\mathcal{B}}_{0}\right) \mathcal{E}_{A}+\left(23+6 \zeta_{2}\right) \mathcal{E}_{F}\right] \\
& \left.+8 \beta_{0} C_{A F}^{2}\left[\left(2 \overline{\mathcal{B}}_{2}+6 \overline{\mathcal{B}}_{1}-5 \overline{\mathcal{B}}_{0}\right) \mathcal{E}_{A}-9 \mathcal{E}_{F}\right]\right\} \\
& +\frac{C_{F} a_{\mathrm{s}}^{3} \beta_{0} \ln ^{3} \tilde{N}}{6 C_{A F}}\left\{C_{F}\left(\beta_{0}-6 C_{F}\right)\left[\overline{\mathcal{B}}_{1} \mathcal{E}_{A}-\mathcal{E}_{F}\right]-3 \beta_{0} C_{F}\left[\overline{\mathcal{B}}_{0} \mathcal{E}_{A}-\mathcal{E}_{F}\right]\right. \\
& \left.+\beta_{0} C_{A F}\left(\overline{\mathcal{B}}_{1}-3 \overline{\mathcal{B}}_{0}\right) \mathcal{E}_{A}+8 C_{A F}^{2} \overline{\mathcal{B}}_{1} \mathcal{E}_{A}+2 C_{F} C_{A F}\left[\overline{\mathcal{B}}_{1} \mathcal{E}_{A}-6 \mathcal{E}_{F}\right]\right\} \\
& -\frac{C_{F} a_{\mathrm{s}}^{4} \beta_{0}^{2} \ln ^{5} \tilde{N}}{9 C_{A F}}\left[C_{A}^{2} \overline{\mathcal{B}}_{0} \mathcal{E}_{A}-C_{F}^{2} \mathcal{E}_{F}\right]
\end{aligned}
$$

with $\mathcal{E}_{A, F}=\exp \left(2 a_{\mathrm{s}} C_{A, F} \ln ^{2} \tilde{N}\right)$. The first term in these results represent the LL contributions, the second to forth terms the NLL corrections, and the rest the new NNLL expressions.

\section{NNLL resummation in semi-inclusive $e^{+} e^{-}$annihilation}

We now address the final-state ('time-like') off-diagonal splitting functions, which have not been presented at NNLL accuracy before, for the NLL expressions see ref. [33]. Since these quantities are closely related to their initial-state ('space-like') counterparts, the formulae for the two cases are very similar. Hence it is convenient to express the time-like results via their difference with respect to the corresponding space-like results as presented in the previous section as

$$
\begin{aligned}
& \frac{N}{n_{f}} P_{\mathrm{qg}}^{\mathrm{T}}\left(N, \alpha_{\mathrm{s}}\right)-\frac{N}{C_{F}} P_{\mathrm{gq}}^{\mathrm{S}}\left(N, \alpha_{\mathrm{s}}\right)=8 a_{\mathrm{s}}^{2} C_{A F} \ln \widetilde{N} \mathcal{B}_{1} \\
& \quad+a_{\mathrm{s}}^{2}\left\{4 a_{\mathrm{s}}\left(6 C_{F}-\beta_{0}\right) C_{A F} \ln ^{2} \widetilde{N} \mathcal{B}_{2}-2\left(24 C_{A F} \zeta_{2}+3 C_{F}-\beta_{0}\right) \mathcal{B}_{1}\right. \\
& \left.\quad+6\left(4 C_{A F} \zeta_{2}+2 C_{F}-\beta_{0}\right) \mathcal{B}_{0}-\beta_{0} \mathcal{B}_{-1}\right\}, \\
& \frac{N}{C_{F}} P_{\mathrm{gq}}^{\mathrm{T}}\left(N, \alpha_{\mathrm{s}}\right)-\frac{N}{n_{f}} P_{\mathrm{qg}}^{\mathrm{S}}\left(N, \alpha_{\mathrm{s}}\right)=8 a_{\mathrm{s}}^{2} C_{A F} \ln \widetilde{N} \overline{\mathcal{B}}_{1} \\
& \quad+a_{\mathrm{s}}^{2}\left\{4 a_{\mathrm{s}}\left(6 C_{F}-\beta_{0}\right) C_{A F} \ln ^{2} \widetilde{N} \overline{\mathcal{B}}_{2}+2\left(24 C_{A F} \zeta_{2}-3 C_{F}+\beta_{0}\right) \overline{\mathcal{B}}_{1}\right. \\
& \left.\quad-2\left(12 C_{A F} \zeta_{2}-6 C_{F}+\beta_{0}\right) \overline{\mathcal{B}}_{0}+\beta_{0} \overline{\mathcal{B}}_{-1}\right\} .
\end{aligned}
$$

The difference between the LL terms of the time-like and space-like splitting functions is zero (after removing the overall leading-order colour factors) [51], the difference of the NLL terms is given by the respective first lines of eqs. (4.1) and (4.2), and the remaining terms represent the difference between the NNLL contributions.

In figure 1 the numerical size of the corrections beyond order $a_{\mathrm{s}}^{3}$ is illustrated at a scale $Q^{2} \simeq M_{Z}^{2}$, where these corrections are entirely dominated by the $a_{\mathrm{s}}^{4}$ terms. The presently known contributions are small, but more large- $N$ terms are needed to arrive at quantitatively reliable results. 

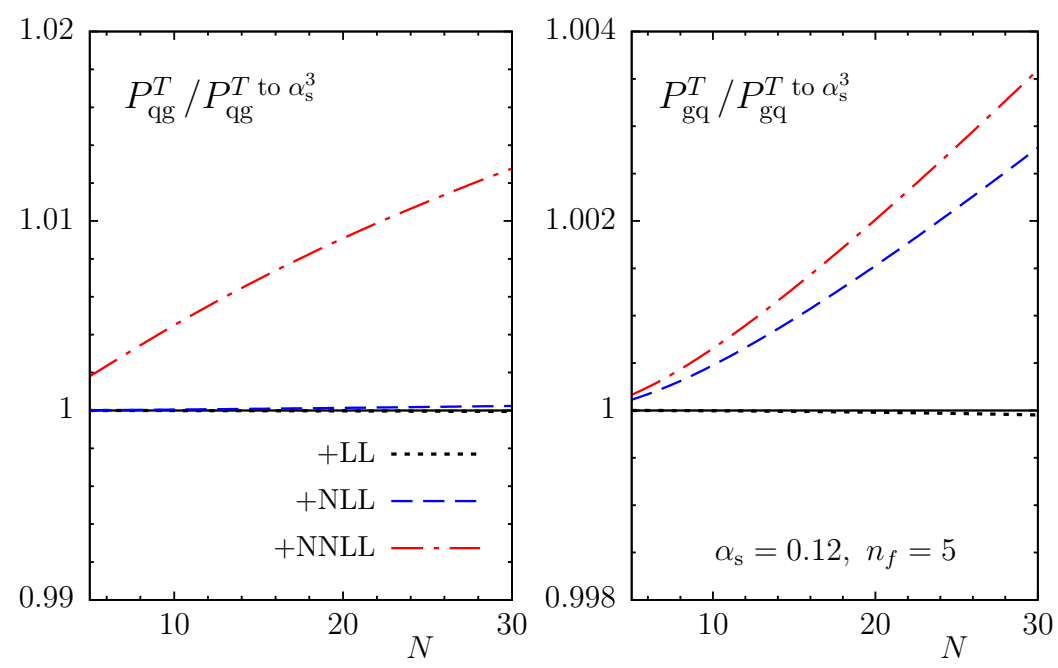

Figure 1. The relative size of the leading-logarithmic (LL), next-to-leading logarithmic (NLL) and next-to-next-to-leading logarithmic (NNLL) higher-order large- $N$ corrections to the NNLO off-diagonal splitting functions $P_{i j}^{T}(N)$ at a typical high-scale reference point.

These $a_{\mathrm{s}}^{4}$ contributions to eqs. (4.1) and (4.2), recall the normalization of $a_{\mathrm{s}}$ in eq. (2.6), read

$$
\begin{aligned}
\frac{1}{n_{f}} P_{\mathrm{qg}}^{T(3)}(x)= & \ln ^{5}(1-x)\left[\frac{22}{27} C_{A F}^{3}-\frac{14}{27} C_{A F}^{2} C_{F}-\frac{4}{27} C_{A F}^{2} n_{f}\right] \\
& +\ln ^{4}(1-x)\left[\left(\frac{1432}{81}+\frac{64}{9} \zeta_{2}\right) C_{A F}^{3}+\left(\frac{1471}{54}-8 \zeta_{2}\right) C_{A F}^{2} C_{F}\right. \\
& \left.-\frac{49}{81} C_{A F} C_{F}^{2}-\frac{16}{3} C_{A F}^{2} n_{f}+\frac{17}{81} C_{A F} C_{F} n_{f}+\frac{32}{81} C_{A F} n_{f}^{2}\right] \\
& +\mathcal{O}\left(\ln ^{3}(1-x)\right) \\
\frac{1}{C_{F}} P_{\mathrm{gq}}^{T}{ }^{(3)}(x)= & \ln ^{5}(1-x)\left[-\frac{26}{27} C_{A F}^{3}-\frac{14}{27} C_{A F}^{2} C_{F}-\frac{4}{27} C_{A F}^{2} n_{f}\right] \\
& +\ln ^{4}(1-x)\left[\left(\frac{469}{27}-\frac{128}{9} \zeta_{2}\right) C_{A F}^{3}+\left(\frac{5317}{162}-8 \zeta_{2}\right) C_{A F}^{2} C_{F}\right. \\
& \left.-\frac{13}{81} C_{A F} C_{F}^{2}-\frac{212}{81} C_{A F}^{2} n_{f}+\frac{17}{81} C_{A F} C_{F} n_{f}-\frac{4}{81} C_{A F} n_{f}^{2}\right] \\
& +\mathcal{O}\left(\ln ^{3}(1-x)\right)
\end{aligned}
$$

after transformation to $x$-space using the second line of eq. (2.3). The corresponding NLO and NNLO expressions can be found in ref. [23]. Note that the coefficients of $\ln ^{6}(1-x)$ are zero for both the space-like and time-like functions. This is due to fact that the LL contributions at order $a_{\mathrm{s}}^{n+1}$ are proportional to the Bernoulli numbers $B_{n}$ which vanishes for all odd values $n>1$. Eqs. (4.3) and (4.4) can be used, e.g., as a check for future fourth-order Feynman diagram calculations of the next-to-next-to-next-to-leading order $\left(\mathrm{N}^{3} \mathrm{LO}\right)$ time-like splitting function or, before, for use with other partial information on these quantities. 
Also for the SIA coefficient functions, the large- $N$ expressions can be conveniently presented via their differences with the corresponding quantities in DIS. In this form the results for transverse and $\phi$-exchange coefficient functions $C_{T, \mathrm{~g}}$ and $C_{\phi, \mathrm{q}}^{\mathrm{T}}$ can be written in a surprisingly compact form,

$$
\begin{aligned}
& \frac{N}{C_{F}} C_{T, \mathrm{~g}}\left(N, \alpha_{\mathrm{s}}\right)-\frac{N}{n_{f}} C_{2, \mathrm{~g}}\left(N, \alpha_{\mathrm{s}}\right)=2 a_{\mathrm{s}} \mathcal{E}_{F} \mathcal{B}_{1} \\
& \quad+\frac{a_{\mathrm{s}}}{4 C_{A F} \ln \widetilde{N}}\left\{\left[\beta_{0}\left(4 \mathcal{B}_{1}-4 \mathcal{B}_{0}+\mathcal{B}_{-1}\right)+12 C_{F}\left(-\mathcal{B}_{1}+\left(1+2 \zeta_{2}\right) \mathcal{B}_{0}\right)\right.\right. \\
& \left.\left.\quad+24 C_{A F} \zeta_{2}\left(2 \mathcal{B}_{1}-\mathcal{B}_{0}\right)\right] \mathcal{E}_{F}-24 C_{A} \zeta_{2} \mathcal{E}_{A}\right\} \\
& \quad-a_{\mathrm{s}}^{2} \ln \tilde{N}\left\{\beta_{0} \mathcal{B}_{2}-6 C_{F}\left(\mathcal{B}_{2}+\mathcal{B}_{1}\right)\right\} \mathcal{E}_{F}+\frac{4}{3} a_{\mathrm{s}}^{3} C_{F} \beta_{0} \ln ^{3} \tilde{N} \mathcal{E}_{F} \mathcal{B}_{1} \\
& \frac{N}{n_{f}} C_{\phi, \mathrm{q}}^{\mathrm{T}}\left(N, \alpha_{\mathrm{s}}\right)-\frac{N}{C_{F}} C_{\phi, \mathrm{q}}\left(N, \alpha_{\mathrm{s}}\right)=-2 a_{\mathrm{s}} \mathcal{E}_{A} \overline{\mathcal{B}}_{1} \\
& \quad+\frac{a_{\mathrm{s}}}{4 C_{A F} \ln \widetilde{N}}\left\{\left[-\beta_{0}\left(4 \overline{\mathcal{B}}_{1}-4 \overline{\mathcal{B}}_{0}-\overline{\mathcal{B}}_{-1}\right)+12 C_{F}\left(\overline{\mathcal{B}}_{1}-\left(1+2 \zeta_{2}\right) \overline{\mathcal{B}}_{0}\right)\right.\right. \\
& \left.\left.\quad+48 C_{A F} \zeta_{2}\left(\overline{\mathcal{B}}_{1}-\overline{\mathcal{B}}_{0}\right)\right] \mathcal{E}_{A}+24 C_{F} \zeta_{2} \mathcal{E}_{F}\right\} \\
& \quad+a_{\mathrm{s}}^{2} \ln \tilde{N}\left\{\beta_{0}\left(\overline{\mathcal{B}}_{2}-2 \overline{\mathcal{B}}_{1}\right)-6 C_{F} \overline{\mathcal{B}}_{2}\right\} \mathcal{E}_{A}-\frac{4}{3} a_{\mathrm{s}}^{3} C_{A} \beta_{0} \ln ^{3} \tilde{N} \mathcal{E}_{A} \overline{\mathcal{B}}_{1} .
\end{aligned}
$$

As for the splitting functions, the difference between the space- and time-like coefficient functions is zero at LL accuracy after dividing out the LO colour factors. The first line of eq. (4.5) is the NLL difference and the remaining terms represent the difference of the NNLL corrections.

The corresponding $x$-space expressions for the coefficient function $C_{T, \mathrm{~g}}$ at order $a_{\mathrm{s}}^{3}$ and $a_{\mathrm{s}}^{4} \mathrm{read}$

$$
\begin{aligned}
\frac{1}{C_{F}} c_{T, \mathrm{~g}}^{(3)}(x)= & \ln ^{5}(1-x)\left[\frac{2}{3} C_{A}^{2}+\frac{10}{3} C_{F}^{2}\right] \\
& +\ln ^{4}(1-x)\left[\frac{7}{27} C_{A} n_{f}-\frac{269}{54} C_{A}^{2}+\frac{17}{27} C_{F} n_{f}-\frac{338}{27} C_{F} C_{A}-\frac{97}{18} C_{F}^{2}\right] \\
& +\ln ^{3}(1-x)\left[\left(\frac{2990}{81}-\frac{16}{9} \zeta_{2}\right) C_{A}^{2}+\left(\frac{3652}{81}-\frac{88}{9} \zeta_{2}\right) C_{F} C_{A}\right. \\
& \left.-\left(\frac{41}{9}+\frac{112}{9} \zeta_{2}\right) C_{F}^{2}-\frac{140}{81} C_{A} n_{f}-\frac{436}{81} C_{F} n_{f}\right] \\
& +\mathcal{O}\left(\ln ^{2}(1-x)\right)
\end{aligned}
$$

a result that has already been presented in ref. [33], and

$$
\begin{aligned}
\frac{1}{C_{F}} c_{T, \mathrm{~g}}^{(4)}(x)= & \ln ^{7}(1-x)\left[\frac{46}{135} C_{A}^{3}+\frac{14}{45} C_{F} C_{A}^{2}-\frac{14}{45} C_{F}^{2} C_{A}+\frac{314}{135} C_{F}^{3}\right] \\
& +\ln ^{6}(1-x)\left[\frac{112}{405} C_{A}^{2} n_{f}-\frac{1696}{405} C_{A}^{3}+\frac{106}{405} C_{F} C_{A} n_{f}-\frac{703}{162} C_{F} C_{A}^{2}\right. \\
& \left.+\frac{502}{405} C_{F}^{2} n_{f}-\frac{5407}{405} C_{F}^{2} C_{A}-\frac{59}{10} C_{F}^{2}\right]
\end{aligned}
$$




$$
\begin{aligned}
& +\ln ^{5}(1-x)\left[\left(\frac{75403}{1620}-\frac{149}{15} \zeta_{2}\right) C_{A}^{3}+\left(\frac{22937}{648}+\frac{52}{15} \zeta_{2}\right) C_{F} C_{A}^{2}\right. \\
& +\left(\frac{10055}{108}-\frac{99}{5} \zeta_{2}\right) C_{F}^{2} C_{A}-\left(\frac{143}{120}+\frac{326}{15} \zeta_{2}\right) C_{F}^{3}+\frac{23}{405} C_{A} n_{f}^{2} \\
& \left.-\frac{521}{135} C_{A}^{2} n_{f}+\frac{97}{405} C_{F} n_{f}^{2}-\frac{3503}{540} C_{F} C_{A} n_{f}-\frac{6013}{540} C_{F}^{2} n_{f}\right] \\
& +\mathcal{O}\left(\ln ^{4}(1-x)\right)
\end{aligned}
$$

The corresponding results for scalar-exchange SIA are given by

$$
\begin{aligned}
\frac{1}{n_{f}} c_{\phi, \mathrm{q}}^{T(3)}(x)= & \ln ^{5}(1-x)\left[\frac{10}{3} C_{A}^{2}+\frac{2}{3} C_{F}^{2}\right] \\
& +\ln ^{4}(1-x)\left[\frac{47}{27} C_{A} n_{f}-\frac{517}{54} C_{A}^{2}+\frac{13}{27} C_{F} n_{f}-\frac{310}{27} C_{F} C_{A}-\frac{55}{6} C_{F}^{2}\right] \\
& +\ln ^{3}(1-x)\left[\left(\frac{6554}{81}-\frac{104}{9} \zeta_{2}\right) C_{A}^{2}+\left(\frac{6139}{81}-\frac{248}{9} \zeta_{2}\right) C_{F} C_{A}\right. \\
& \left.+\left(\frac{64}{3}+\frac{208}{9} \zeta_{2}\right) C_{F}^{2}+\frac{16}{27} n_{f}^{2}-\frac{1268}{81} C_{A} n_{f}-\frac{970}{81} C_{F} n_{f}\right] \\
& +\mathcal{O}\left(\ln ^{2}(1-x)\right), \\
\frac{1}{n_{f} c_{\phi, \mathrm{q}}^{T(4)}(x)=} & \ln ^{7}(1-x)\left[\frac{314}{135} C_{A}^{3}-\frac{14}{45} C_{F} C_{A}^{2}+\frac{14}{45} C_{F}^{2} C_{A}+\frac{46}{135} C_{F}^{3}\right] \\
& +\ln ^{6}(1-x)\left[\frac{1004}{405} C_{A}^{2} n_{f}-\frac{5522}{405} C_{A}^{3}+\frac{2}{405} C_{F} C_{A} n_{f}-\frac{6403}{810} C_{F} C_{A}^{2}\right. \\
& \left.+\frac{254}{405} C_{F}^{2} n_{f}-\frac{2171}{405} C_{F}^{2} C_{A}-\frac{559}{90} C_{F}^{3}\right] \\
& +\ln ^{5}(1-x)\left[\left(\frac{194611}{1620}-\frac{1183}{45} \zeta_{2}\right) C_{A}^{3}+\left(\frac{16873}{216}-\frac{796}{45} \zeta_{2}\right) C_{F} C_{A}^{2}\right. \\
& +\left(\frac{103781}{1620}-\frac{499}{45} \zeta_{2}\right) C_{F}^{2} C_{A}+\left(\frac{9649}{360}+\frac{226}{15} \zeta_{2}\right) C_{F}^{3}+\frac{187}{135} C_{A} n_{f}^{2} \\
& \left.-\frac{10846}{405} C_{A}^{2} n_{f}+\frac{53}{135} C_{F} n_{f}^{2}-\frac{709}{60} C_{F} C_{A} n_{f}-\frac{20993}{1620} C_{F}^{2} n_{f}\right] \\
& +\mathcal{O}\left(\ln ^{4}(1-x)\right) \cdot \\
& \\
&
\end{aligned}
$$

As for $C_{2, q}, C_{T, q}$ and $C_{\phi, g}^{(T)}$, the leading (and subleading) $1 / N^{k}$ parts of the DIS and SIA quark coefficient functions for $F_{L}$ are given by 'non-singlet' contributions that have been derived and discussed in refs. [36, 37]. The gluon coefficient functions have an analytical structure analogous to eqs. (4.1) and (4.5), with the SIA (time-like, T) and DIS large- $N$ expressions for $C_{L, g}$ differing only at NNLL accuracy. The same holds for $C_{L, q}$, 
see eq. (6.16) of ref. [37]. We find

$$
\frac{N^{2}}{C_{F}} C_{L, \mathrm{~g}}^{\mathrm{T}}\left(N, \alpha_{\mathrm{s}}\right)-\frac{N^{2}}{2 n_{f}} C_{L, \mathrm{~g}}\left(N, \alpha_{\mathrm{s}}\right)=4 a_{\mathrm{s}}^{2} C_{F} \mathcal{E}_{F} \mathcal{B}_{1}+48 a_{\mathrm{s}}^{2} C_{A} \zeta_{2} \mathcal{E}_{A}
$$

where the analytic NNLL expression for $C_{L, \mathrm{~g}}$ has already been given in eq. (6.3) of ref. [32].

The resulting third- and fourth-order NNLL threshold expansion of $C_{L, g}^{T}$ in $x$-space is given by

$$
\begin{aligned}
(1-x)^{-1} c_{L, \mathrm{~g}}^{T(3)}(x)= & 8 C_{F} C_{A}^{2} \ln ^{4}(1-x) \\
& +\ln ^{3}(1-x) C_{F}\left[\frac{20}{3} C_{F}^{2}+\frac{52}{3} C_{F} C_{A}-\frac{952}{9} C_{A}^{2}+\frac{16}{9} C_{A} n_{f}\right] \\
& +\ln ^{2}(1-x) C_{F}\left[\left(62-32 \zeta_{2}\right) C_{F}^{2}-\left(\frac{784}{3}-32 \zeta_{2}\right) C_{A} C_{F}+\frac{5720}{9} C_{A}^{2}\right. \\
& \left.-\frac{224}{9} C_{A} n_{f}+\frac{16}{3} C_{F} n_{f}-64 n_{f}^{2} \frac{d^{a b c} d_{a b c}}{n_{a}} f l_{11}^{g}\left(11+2 \zeta_{2}-12 \zeta_{3}\right)\right] \\
& +\mathcal{O}(\ln (1-x)), \\
(1-x)^{-1} c_{L, \mathrm{~g}}^{T(4)}(x)= & \frac{16}{3} C_{F} C_{A}^{3} \ln ^{6}(1-x) \\
& +\ln ^{5}(1-x) C_{F}\left[\frac{20}{3} C_{F}^{3}+\frac{52}{3} C_{F} C_{A}^{2}-\frac{1040}{9} C_{A}^{3}+\frac{32}{9} C_{A}^{2} n_{f}\right] \\
& +\ln ^{4}(1-x) C_{F}\left[\left(\frac{323}{9}-\frac{160}{3} \zeta_{2}\right) C_{F}^{3}+\left(\frac{536}{27}+16 \zeta_{2}\right) C_{A} C_{F}^{2}\right. \\
& -\left(\frac{12629}{27}-\frac{160}{3} \zeta_{2}\right) C_{F} C_{A}^{2}+\left(\frac{35380}{27}-80 \zeta_{2}\right) C_{A}^{3} \\
& +\frac{154}{27} C_{F}^{2} n_{f}+\frac{278}{27} C_{F} C_{A} n_{f}-\frac{2096}{27} C_{A}^{2} n_{f}+\frac{16}{27} C_{A} n_{f}^{2} \\
& \left.-128 n_{f}^{2} \frac{d^{a b c} d_{a b c}}{n_{a}} f l_{11}^{g} C_{A}\left(11+2 \zeta_{2}-12 \zeta_{3}\right)\right] \\
& +\mathcal{O}\left(\ln ^{3}(1-x)\right) .
\end{aligned}
$$

Eq. (4.13) was already given in ref. [33] if, for brevity, without the $f l_{11}^{g}$ contribution (see figure 1 of ref. [6] for a typical DIS diagram contributing to this flavour structure) which, for photon-exchange SIA, corresponds to the charge factor

$$
f l_{11}^{\mathrm{g}}=\langle e\rangle^{2} /\left\langle e^{2}\right\rangle \quad \text { with } \quad\left\langle e^{k}\right\rangle=n_{f}^{-1} \sum_{i=1}^{n_{f}} e_{i}^{k},
$$

where $e_{i}$ is the charge of the $i$-th effectively massless flavour in units of the proton charge. Analogous terms also contribute to $C_{T, g}$ at the same powers of $\ln (1-x)$, but there these logarithms are below NNLL accuracy, recall eqs. (2.14) and (2.15). Note that different normalizations are used in the literature for the QCD group factor $d^{a b c} d_{a b c} / n_{a}=3 / 8 d^{a b c} d_{a b c} / n_{c}=$ $5 / 48$. Also note that our normalization of both functions (as that of other recent articles) 

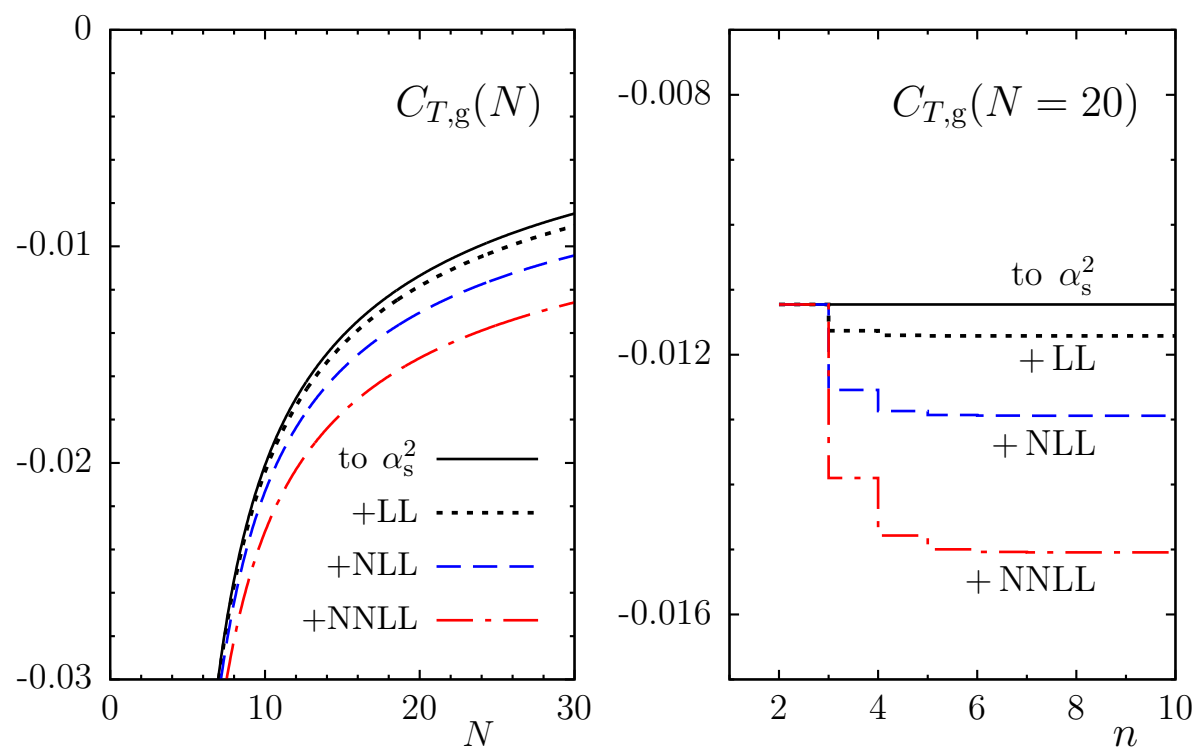

Figure 2. Left: the large- $N$ behaviour of the second-order and resummed $\overline{\mathrm{MS}}$-scheme gluon coefficient function for the transverse fragmentation function at a high-scale reference point $\alpha_{\mathrm{s}}=$ 0.12 for $n_{f}=5$ light quark flavours. Right: the LL, NLL and NNLL contributions of the third to the tenth orders in $\alpha_{\mathrm{s}}$, added at the corresponding values of the abscissa, to those results at $N=20$.

differs by a factor of $\frac{1}{2}$ from that of refs. $[8,9,11]$, i.e., here the first-order large- $x$ limits read

$$
C_{T, g}\left(x, a_{\mathrm{s}}\right)=2 C_{F} a_{\mathrm{s}} \ln (1-x)+\ldots \text { and } C_{L, g}^{T}\left(x, a_{\mathrm{s}}\right)=4 C_{F} a_{\mathrm{s}}(1-x)+\ldots
$$

The results for $C_{T, g}$ in eq. (4.5) and for $C_{L, g}^{T}$ in eq. (4.11) are illustrated at the standard high-scale reference point $Q^{2} \simeq M_{Z}^{2}$ (recall that we identify the renormalization and factorization scales with $Q^{2}$ throughout this article) in figure 2 and figure 3, respectively. As for the splitting function in figure 1, the LL terms have a small numerical effect. However, the overall (relative) size of the corrections - note that the effect of these coefficient functions is much smaller than that of their quark counterparts $[36,37]$ - is large here, and contributions beyond order $a_{\mathrm{s}}^{4}$ are not negligible as shown for one moment $N$ in the right panels. Clearly higher terms in the large- $N$ expansion, or other information complementing our results, are required to quantitatively establish the size of the higher-order large- $N$ contributions to $C_{T, g}$ and $C_{L, g}^{T}$. The results for $C_{\phi, q}^{T}$ in eq. (4.6) are similar but not shown here, since this quantity is of mainly theoretical importance.

\section{Summary and outlook}

Considerable progress has been made in the past seven years on the resummation of large- $x$ (or, in Mellin space, large- $N$ ) threshold logarithms [32-51] beyond those addressed by the soft-gluon exponentiation (SGE) [25-29]. This holds for sub-leading contributions, in terms of powers of $(1-x)$ or $1 / N$ for $x \rightarrow 1$ or $N \rightarrow \infty$, to quantities to which the SGE is applicable for the leading terms, as well as for which the SGE is not applicable at all. 

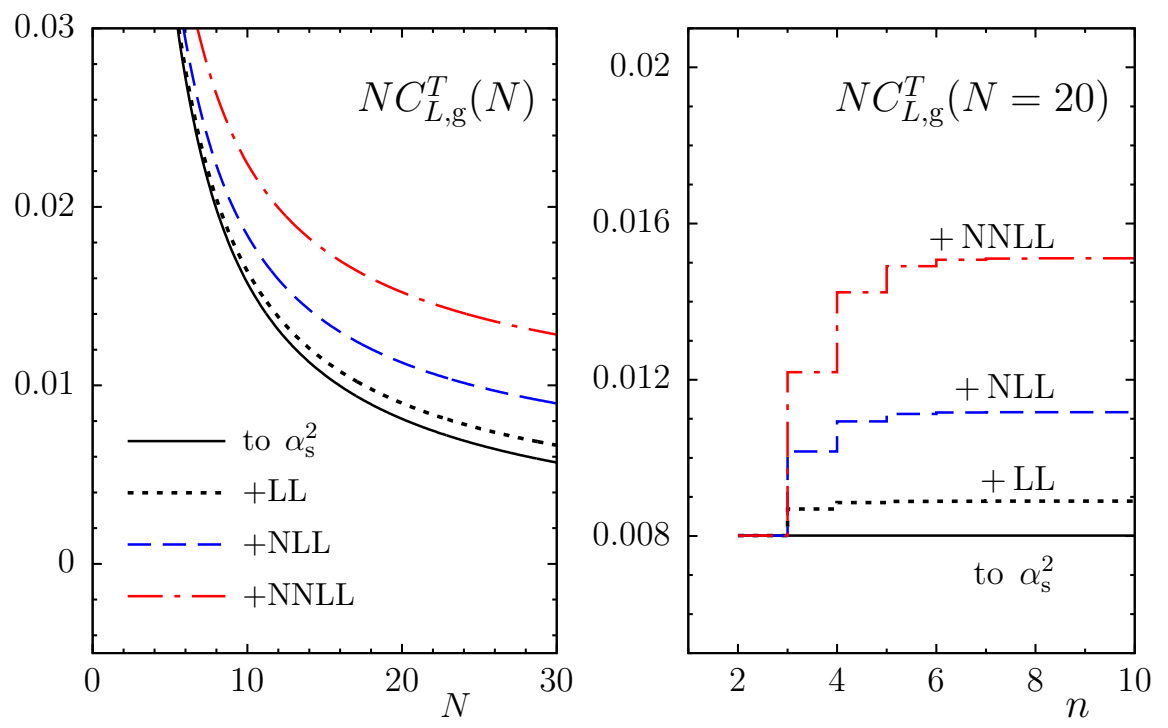

Figure 3. As figure 2, but for the gluon coefficient function of the longitudinal fragmentation function in photon-exchange SIA. All curves have been multiplied by $N$ for display purposes.

So far most of the explicit large- $x$ results for higher-order splitting functions and coefficient functions have been obtained by studying physical evolution kernels [36-40, 48-50] and the structure of unfactorized cross sections in dimensional regularization [32-35, 51] (see refs. [53, 54] for an analogous small- $x$ resummation in SIA). The former approach is particularly suited for non-singlet quantities, i.e., quantities that only involve the quarkquark and gluon-gluon splitting functions which are stable in the threshold limit [17-22] in the standard $\overline{\mathrm{MS}}$ factorization scheme adopted throughout this article. Where applicable beyond the leading logarithms, presently for inclusive deep-inelastic scattering (DIS) and semi-inclusive $e^{+} e^{-}$annihilation (SIA), the resummation via unfactorized cross sections leads to the same results but is slightly more powerful even in those non-singlet cases. The resummation of the off-diagonal quark-gluon and gluon-quark splitting function is possible only in this second approach.

Both methods are, so far, less effective for lepton-pair and Higgs-boson production, the former due to the lack of suitable flavour-singlet physical evolution kernels, the latter due to the different phase-space structure of these hadron collider processes. However, the complete analogy of the non-singlet physical kernels for SIA, DIS, the Drell-Yan process and Higgs production [37, 40] suggests that there is presently unearthed resummation information also in the unfactorized expressions for the latter two processes.

In the present article, we have first reconsidered the NNLL threshold resummation of the flavour-singlet structure functions in DIS. This resummation was performed already in ref. [32] where, however, a closed NNLL expression was given only for the simplest case, the longitudinal structure function $F_{L}$. Here we have presented corresponding closed forms also for the off-diagonal splitting functions $P_{q g}$ and $P_{g q}$ for the evolution of the parton distributions of hadrons and for the corresponding coefficient functions for $F_{2}$ and $F_{\phi}$, where the latter occurs in DIS via Higgs-boson exchange in the heavy-top limit. 
We have then extended this resummation to the theoretically closely related, see ref. [21-23], case of SIA and presented the NNLL resummation of the 'time-like' splitting functions $P_{q g}^{T}$ and $P_{g q}^{T}$ for the evolution of final-state fragmentation distributions and the corresponding coefficient functions for flavour-singlet fragmentation functions. All these results can be expressed in terms of the Bernoulli functions introduced in ref. [51] together with, for the coefficient functions, the quark and gluon leading-logarithmic soft-gluon exponentials. As already in ref. $[32,51]$, the analysis of physical kernels was useful for finding the rather complicated closed NNLL expressions for the coefficient functions.

We find that the resummation of the highest three logarithms, which leads to small corrections for the off-diagonal splitting functions but large effects for the corresponding coefficient functions, does not lead to numerically reliable results: the LL terms are always small, and the NLL contributions mostly smaller than the NNLL 'corrections'; a situation that, in fact, is similar to that in the SGE of the corresponding diagonal quantities refs. [30, 31]. Yet we expect our results to become phenomenologically useful in connection with other efforts such as, e.g., the extension of the fourth-order calculations of ref. [55-58] to higher moments and other quantities. Furthermore one may hope that the wealth of results derived here and before can provide some assistance to extending the reach of the non-SGE threshold resummation to higher logarithmic accuracies.

\section{Acknowledgments}

This research has been supported by the German Research Foundation (DFG) through Sonderforschungsbereich Transregio 9, Computergestützte Theoretische Teilchenphysik, the Schweizer Nationalfonds (SNF) under grant 200020-162487 and the U.K. Science \& Technology Facilities Council (STFC) under grant numbers ST/J000493/1 and ST/L000431/1. Our calculations were performed using the symbolic manipulation system FORM [59-61].

Open Access. This article is distributed under the terms of the Creative Commons Attribution License (CC-BY 4.0), which permits any use, distribution and reproduction in any medium, provided the original author(s) and source are credited.

\section{References}

[1] Particle Data Group collaboration, K.A. Olive et al., Review of Particle Physics, Chin. Phys. C 38 (2014) 090001 [inSPIRE].

[2] W.L. van Neerven and E.B. Zijlstra, Order $\alpha_{S}^{2}$ contributions to the deep inelastic Wilson coefficient, Phys. Lett. B 272 (1991) 127 [InSPIRE].

[3] E.B. Zijlstra and W.L. van Neerven, Contribution of the second order gluonic Wilson coefficient to the deep inelastic structure function, Phys. Lett. B 273 (1991) 476 [INSPIRE].

[4] E.B. Zijlstra and W.L. van Neerven, Order $\alpha_{S}^{2}$ correction to the structure function $F_{3}\left(x, Q^{2}\right)$ in deep inelastic neutrino-hadron scattering, Phys. Lett. B 297 (1992) 377 [INSPIRE].

[5] S. Moch and J.A.M. Vermaseren, Deep inelastic structure functions at two loops, Nucl. Phys. B 573 (2000) 853 [hep-ph/9912355] [INSPIRE]. 
[6] J.A.M. Vermaseren, A. Vogt and S. Moch, The Third-order QCD corrections to deep-inelastic scattering by photon exchange, Nucl. Phys. B 724 (2005) 3 [hep-ph/0504242] [INSPIRE].

[7] S. Moch, J.A.M. Vermaseren and A. Vogt, Third-order QCD corrections to the charged-current structure function $F_{3}$, Nucl. Phys. B 813 (2009) 220 [arXiv:0812.4168].

[8] P.J. Rijken and W.L. van Neerven, $O\left(\alpha_{S}^{2}\right)$ contributions to the longitudinal fragmentation function in $e^{+} e^{-}$annihilation, Phys. Lett. B 386 (1996) 422 [hep-ph/9604436] [INSPIRE].

[9] P.J. Rijken and W.L. van Neerven, Higher order QCD corrections to the transverse and longitudinal fragmentation functions in electron-positron annihilation, Nucl. Phys. B 487 (1997) 233 [hep-ph/9609377] [InSPIRE].

[10] P.J. Rijken and W.L. van Neerven, $O\left(\alpha_{S}^{2}\right)$ contributions to the asymmetric fragmentation function in $e^{+} e^{-}$annihilation, Phys. Lett. B 392 (1997) 207 [hep-ph/9609379] [INSPIRE].

[11] A. Mitov and S.-O. Moch, QCD Corrections to Semi-Inclusive Hadron Production in Electron-Positron Annihilation at Two Loops, Nucl. Phys. B 751 (2006) 18 [hep-ph/0604160] [INSPIRE].

[12] R.V. Harlander and W.B. Kilgore, Next-to-next-to-leading order Higgs production at hadron colliders, Phys. Rev. Lett. 88 (2002) 201801 [hep-ph/0201206] [INSPIRE].

[13] C. Anastasiou and K. Melnikov, Higgs boson production at hadron colliders in NNLO QCD, Nucl. Phys. B 646 (2002) 220 [hep-ph/0207004] [INSPIRE].

[14] V. Ravindran, J. Smith and W.L. van Neerven, NNLO corrections to the total cross-section for Higgs boson production in hadron hadron collisions, Nucl. Phys. B 665 (2003) 325 [hep-ph/0302135] [INSPIRE].

[15] C. Anastasiou, C. Duhr, F. Dulat, F. Herzog and B. Mistlberger, Higgs Boson Gluon-Fusion Production in QCD at Three Loops, Phys. Rev. Lett. 114 (2015) 212001 [arXiv:1503.06056] [INSPIRE].

[16] C. Anzai et al., Exact $N^{3} L O$ results for $q q^{\prime} \rightarrow H+X$, JHEP 07 (2015) 140 [arXiv: 1506.02674] [INSPIRE].

[17] G.P. Korchemsky, Asymptotics of the Altarelli-Parisi-Lipatov Evolution Kernels of Parton Distributions, Mod. Phys. Lett. A 4 (1989) 1257 [INSPIRE].

[18] Yu. L. Dokshitzer, G. Marchesini and G.P. Salam, Revisiting parton evolution and the large-x limit, Phys. Lett. B 634 (2006) 504 [hep-ph/0511302] [INSPIRE].

[19] S. Moch, J.A.M. Vermaseren and A. Vogt, The Three loop splitting functions in QCD: The Nonsinglet case, Nucl. Phys. B 688 (2004) 101 [hep-ph/0403192] [InSPIRE].

[20] A. Vogt, S. Moch and J.A.M. Vermaseren, The Three-loop splitting functions in QCD: The Singlet case, Nucl. Phys. B 691 (2004) 129 [hep-ph/0404111] [INSPIRE].

[21] A. Mitov, S. Moch and A. Vogt, Next-to-Next-to-Leading Order Evolution of Non-Singlet Fragmentation Functions, Phys. Lett. B 638 (2006) 61 [hep-ph/0604053] [INSPIRE].

[22] S. Moch and A. Vogt, On third-order timelike splitting functions and top-mediated Higgs decay into hadrons, Phys. Lett. B 659 (2008) 290 [arXiv:0709.3899] [INSPIRE].

[23] A.A. Almasy, S. Moch and A. Vogt, On the Next-to-Next-to-Leading Order Evolution of Flavour-Singlet Fragmentation Functions, Nucl. Phys. B 854 (2012) 133 [arXiv:1107.2263] [INSPIRE]. 
[24] S. Moch, J.A.M. Vermaseren and A. Vogt, The Three-Loop Splitting Functions in QCD: The Helicity-Dependent Case, Nucl. Phys. B 889 (2014) 351 [arXiv:1409.5131] [InSPIRE].

[25] G.F. Sterman, Summation of Large Corrections to Short Distance Hadronic Cross-Sections, Nucl. Phys. B 281 (1987) 310 [inSPIRE].

[26] S. Catani and L. Trentadue, Resummation of the QCD Perturbative Series for Hard Processes, Nucl. Phys. B 327 (1989) 323 [InSPIRE].

[27] L. Magnea, All Order Summation and Two Loop Results for the Drell-Yan Cross-section, Nucl. Phys. B 349 (1991) 703 [inSPIRE].

[28] S. Catani, M.L. Mangano, P. Nason and L. Trentadue, The Resummation of soft gluons in hadronic collisions, Nucl. Phys. B 478 (1996) 273 [hep-ph/9604351] [INSPIRE].

[29] H. Contopanagos, E. Laenen and G.F. Sterman, Sudakov factorization and resummation, Nucl. Phys. B 484 (1997) 303 [hep-ph/9604313] [INSPIRE].

[30] S. Moch, J.A.M. Vermaseren and A. Vogt, Higher-order corrections in threshold resummation, Nucl. Phys. B 726 (2005) 317 [hep-ph/0506288] [INSPIRE].

[31] S. Moch and A. Vogt, Higher-order threshold resummation for semi-inclusive $e^{+} e^{-}$ annihilation, Phys. Lett. B 680 (2009) 239 [arXiv:0908.2746] [INSPIRE].

[32] A.A. Almasy, G. Soar and A. Vogt, Generalized double-logarithmic large-x resummation in inclusive deep-inelastic scattering, JHEP 03 (2011) 030 [arXiv:1012.3352] [INSPIRE].

[33] N.A. Lo Presti, A. Vogt and A.A. Almasy, Generalized Threshold Resummation for Semi-Inclusive $e^{+} e^{-}$Annihilation, arXiv:1202.5224 [INSPIRE].

[34] A. Vogt et al., Progress on double-logarithmic large-x and small-x resummations for (semi-)inclusive hard processes, arXiv:1212.2932 [INSPIRE].

[35] N.A. Lo Presti, A.A. Almasy and A. Vogt, Leading large-x logarithms of the quark-gluon contributions to inclusive Higgs-boson and lepton-pair production, Phys. Lett. B 737 (2014) 120 [arXiv: 1407.1553] [INSPIRE].

[36] S. Moch and A. Vogt, Threshold Resummation of the Structure Function $F(L)$, JHEP 04 (2009) 081 [arXiv:0902.2342] [INSPIRE].

[37] S. Moch and A. Vogt, On non-singlet physical evolution kernels and large-x coefficient functions in perturbative QCD, JHEP 11 (2009) 099 [arXiv:0909.2124] [INSPIRE].

[38] G. Soar, S. Moch, J.A.M. Vermaseren and A. Vogt, On Higgs-exchange DIS, physical evolution kernels and fourth-order splitting functions at large $x$, Nucl. Phys. B 832 (2010) 152 [arXiv:0912.0369] [INSPIRE].

[39] A. Vogt, G. Soar, S. Moch and J.A.M. Vermaseren, On higher-order flavour-singlet splitting and coefficient functions at large $x, P o S$ DIS2010 (2010) 139 [arXiv:1008.0952] [INSPIRE].

[40] D. de Florian, J. Mazzitelli, S. Moch and A. Vogt, Approximate $N^{3}$ LO Higgs-boson production cross section using physical-kernel constraints, JHEP 10 (2014) 176 [arXiv: 1408.6277] [INSPIRE].

[41] E. Laenen, L. Magnea and G. Stavenga, On next-to-eikonal corrections to threshold resummation for the Drell-Yan and DIS cross sections, Phys. Lett. B 669 (2008) 173 [arXiv: 0807.4412] [INSPIRE].

[42] E. Laenen, G. Stavenga and C.D. White, Path integral approach to eikonal and next-to-eikonal exponentiation, JHEP 03 (2009) 054 [arXiv: 0811.2067] [INSPIRE]. 
[43] E. Gardi, E. Laenen, G. Stavenga and C.D. White, Webs in multiparton scattering using the replica trick, JHEP 11 (2010) 155 [arXiv:1008.0098] [INSPIRE].

[44] E. Laenen, L. Magnea, G. Stavenga and C.D. White, Next-to-eikonal corrections to soft gluon radiation: a diagrammatic approach, JHEP 01 (2011) 141 [arXiv:1010.1860] [INSPIRE].

[45] C.D. White, Diagrammatic insights into next-to-soft corrections, Phys. Lett. B 737 (2014) 216 [arXiv:1406.7184] [INSPIRE].

[46] D. Bonocore, E. Laenen, L. Magnea, L. Vernazza and C.D. White, The method of regions and next-to-soft corrections in Drell-Yan production, Phys. Lett. B 742 (2015) 375 [arXiv: 1410.6406] [INSPIRE].

[47] D. Bonocore, E. Laenen, L. Magnea, S. Melville, L. Vernazza and C.D. White, A factorization approach to next-to-leading-power threshold logarithms, JHEP 06 (2015) 008 [arXiv: 1503.05156] [INSPIRE].

[48] G. Grunberg and V. Ravindran, On threshold resummation beyond leading 1-x order, JHEP 10 (2009) 055 [arXiv:0902.2702] [INSPIRE].

[49] G. Grunberg, Large-x structure of physical evolution kernels in Deep Inelastic Scattering, Phys. Lett. B 687 (2010) 405 [arXiv:0911.4471] [INSPIRE].

[50] G. Grunberg, On threshold resummation of singlet structure and fragmentation functions, Nucl. Phys. B 851 (2011) 30 [arXiv:1101.5377] [INSPIRE].

[51] A. Vogt, Leading logarithmic large-x resummation of off-diagonal splitting functions and coefficient functions, Phys. Lett. B 691 (2010) 77 [arXiv:1005.1606] [INSPIRE].

[52] M. Abramowitz and I.A. Stegun eds., Handbook of Mathematical Functions, Dover, New York U.S.A. (1965).

[53] A. Vogt, Resummation of small-x double logarithms in QCD: semi-inclusive electron-positron annihilation, JHEP 10 (2011) 025 [arXiv: 1108.2993] [INSPIRE].

[54] C.H. Kom, A. Vogt and K. Yeats, Resummed small-x and first-moment evolution of fragmentation functions in perturbative QCD, JHEP 10 (2012) 033 [arXiv:1207.5631] [INSPIRE].

[55] P.A. Baikov and K.G. Chetyrkin, New four loop results in $Q C D$, Nucl. Phys. Proc. Suppl. 160 (2006) 76 [INSPIRE].

[56] V.N. Velizhanin, Four loop anomalous dimension of the second moment of the non-singlet twist-2 operator in QCD, Nucl. Phys. B 860 (2012) 288 [arXiv:1112.3954] [INSPIRE].

[57] V.N. Velizhanin, Four loop anomalous dimension of the third and fourth moments of the non-singlet twist-2 operator in QCD, arXiv:1411.1331 [INSPIRE].

[58] P.A. Baikov, K.G. Chetyrkin and J.H. Kühn, Massless Propagators, R(s) and Multiloop QCD, Nucl. Part. Phys. Proc. 261-262 (2015) 3 [arXiv:1501.06739] [INSPIRE].

[59] J.A.M. Vermaseren, New features of FORM, math-ph/0010025 [INSPIRE].

[60] M. Tentyukov and J.A.M. Vermaseren, The Multithreaded version of FORM, Comput. Phys. Commun. 181 (2010) 1419 [hep-ph/0702279] [INSPIRE].

[61] J. Kuipers, T. Ueda, J.A.M. Vermaseren and J. Vollinga, FORM version 4.0, Comput. Phys. Commun. 184 (2013) 1453 [arXiv:1203.6543] [INSPIRE]. 